\title{
Time-Varying MIMO Channels: Parametric Statistical Modeling and Experimental Results
}

Michael A. Jensen

jensen@byu.edu

Shuangquan Wang

Ali Abdi

Jari Salo

Hassan M. El-Sallabi

See next page for additional authors

Follow this and additional works at: https://scholarsarchive.byu.edu/facpub

Part of the Electrical and Computer Engineering Commons

\section{Original Publication Citation}

Shuangquan Wang, et al. "Time-Varying MIMO Channels: Parametric Statistical Modeling and Experimental Results." Vehicular Technology, IEEE Transactions on 56.4 (27): 1949-63

\section{BYU ScholarsArchive Citation}

Jensen, Michael A.; Wang, Shuangquan; Abdi, Ali; Salo, Jari; El-Sallabi, Hassan M.; Wallace, Jon W.; and Vainikainen, Pertti, "Time-Varying MIMO Channels: Parametric Statistical Modeling and Experimental Results" (2007). Faculty Publications. 246.

https://scholarsarchive.byu.edu/facpub/246

This Peer-Reviewed Article is brought to you for free and open access by BYU ScholarsArchive. It has been accepted for inclusion in Faculty Publications by an authorized administrator of BYU ScholarsArchive. For more information, please contact ellen_amatangelo@byu.edu. 


\section{Authors}

Michael A. Jensen, Shuangquan Wang, Ali Abdi, Jari Salo, Hassan M. El-Sallabi, Jon W. Wallace, and Pertti Vainikainen 


\section{Time-Varying MIMO Channels: Parametric Statistical Modeling and Experimental Results}

Shuangquan Wang, Student Member, IEEE, Ali Abdi, Member, IEEE, Jari Salo, Hassan M. El-Sallabi, Member, IEEE, Jon W. Wallace, Member, IEEE, Pertti Vainikainen, Member, IEEE, and Michael A. Jensen, Senior Member, IEEE

\begin{abstract}
Accurate characterization of multiple-input multiple-output (MIMO) fading channels is an important prerequisite for the design of multiantenna wireless-communication systems. In this paper, a single-bounce two-ring statistical model for the time-varying MIMO flat fading channels is proposed. In the model, both the base and mobile stations are surrounded by their own ring of scatterers. For the proposed model, a closed-form expression for the spatio-temporal cross-correlational function between any two subchannels is derived, assuming single-bounce scattering. The new analytical expression includes several key physical parameters of interest such as the mean angle-ofdeparture, the mean angle-of-arrival, the associated angle spreads, and the Doppler spread in a compact form. The model includes many existing correlation models as special cases. Its utility is demonstrated by a comparison with collected MIMO data in terms of the spatio-temporal correlations, level crossing rate, average fade duration, and the instantaneous mutual information.
\end{abstract}

Index Terms-Average fade duration (AFD), channel modeling, instantaneous mutual information (IMI), level crossing rate (LCR), multiantenna systems, multiple input multiple output (MIMO), Rayleigh fading, spatio-temporal cross correlation (STCC).

\section{INTRODUCTION}

$\mathbf{T}$ HE UTILIZATION of antenna arrays at the base station (BS) and the mobile station (MS) in a wirelesscommunication system increases the capacity linearly with $\min \left(N_{\mathrm{T}}, N_{\mathrm{R}}\right)$, where $N_{\mathrm{T}}$ and $N_{\mathrm{R}}$ are the numbers of transmit

Manuscript received October 27, 2005; revised June 2, 2006; accepted July 20, 2006. This paper was presented in part at the IEEE Sarnoff Symposium on Advances in Wired and Wireless Communications, Princeton, NJ, 2004 [1], and the IEEE International Workshop on Signal Processing Advances for Wireless Communications, New York, NY, 2005 [2]. The review of this paper was coordinated by Dr. K. Dandekar.

S. Wang was with the Department of Electrical and Computer Engineering, New Jersey Institute of Technology, Newark, NJ 07102 USA. He is now with NEC Laboratories America, Inc., Princeton, NJ 08540 USA (e-mail: shuangquan.wang@njit.edu; shuangquan@nec-labs.com).

A. Abdi is with the Department of Electrical and Computer Engineering, New Jersey Institute of Technology, Newark, NJ 07102 USA (e-mail: ali. abdi@njit.edu).

J. Salo is with the European Communications Engineering Ltd., 02150 Espoo, Finland.

H. M. El-Sallabi is with the Information Systems Laboratory, Stanford University, Stanford, CA 94305 USA.

J. W. Wallace is with the International University Bremen, 28725 Bremen, Germany.

P. Vainikainen is with the Radio Laboratory, Helsinki University of Technology, 02015-TKK Espoo, Finland.

M. A. Jensen is with the Department of Electrical and Computer Engineering, Brigham Young University, Provo, UT 84602 USA.

Color versions of one or more of the figures in this paper are available online at http://ieeexplore.ieee.org.

Digital Object Identifier 10.1109/TVT.2007.897643
(Tx) and receive (Rx) antenna elements, respectively, provided that the environment is sufficiently rich in multipath components [3], [4]. The significant increase of channel capacity was originally reported for uncorrelated subchannels ${ }^{1}$ [3], [4]. However, later, it was realized that the correlation among subchannels can significantly affect capacity [5]-[8] and other system performance metrics [9]-[12]. For an overview of multipleinput multiple-output (MIMO) channel models, see [13]-[16] and the references therein. In this paper, we concentrate on the statistical modeling of time-varying MIMO flat $^{2}$ fading channels and verify the utility of the proposed model via comparison with different sets of data.

In a typical macrocell, the BS is elevated and receives the signal within a narrow beamwidth, whereas the MS is surrounded by local scatterers. MIMO modeling of this typical macrocell environment is investigated in [5], where a closed-form expression for the MIMO spatio-temporal cross correlation (STCC) is derived, assuming nonisotropic scattering around the MS. However, in the outdoor and indoor picocells, both the BS and MS are normally surrounded by local scatterers. Clearly, the MIMO macrocell model of [5] cannot be used for such environments. For this situation, we need a double-directional channel model (see [17] and [18], where the double-directional concept is introduced, and some measurements results are provided).

In this paper, the space-time model of [5] is extended by adding another ring of scatterers around the BS in order to facilitate a new and mathematically tractable MIMO STCC model that is needed for analytical calculations and system design, for example, array optimization [19]. We call the newly extended model as the single-bounce two-ring model since only single-bounce rays are considered. The proposed model includes [5] and some other existing correlation models as special cases. Note that the proposed model belongs to the class of double-directional channel models since it includes angular information at both the BS and MS. Most importantly, the proposed parametric model provides a compact analytical form for MIMO STCC in terms of several key physical parameters of the channel. It also avoids the technical difficulties of the double-bounce two-ring model (e.g., [20]), which is discussed in [13]. The parametric nature of the model makes it adaptable to a variety of propagation environments, and its

\footnotetext{
${ }^{1}$ In this paper, each subchannel represents the radio link between each Tx/Rx pair of antennas with the time-varying gain in (2).

${ }^{2}$ Only the flat fading is considered here since the MIMO frequency-selective fading channel can be converted into several flat fading channels by the orthogonal frequency-division-multiplexing (OFDM) technique.
} 


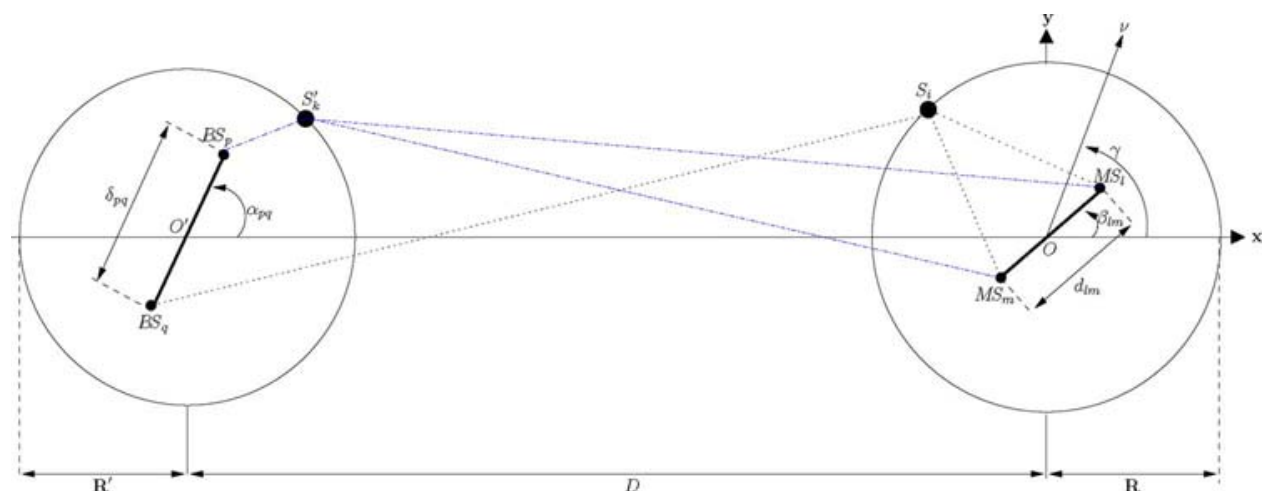

Fig. 1. Geometrical configuration of a $2 \times 2$ channel with local scatterers around the MS and BS, with some single-bounce rays shown in the forward channel.

compact mathematical form is convenient for both analytical studies and numerical calculations. Finally, comparison of the model with the data collected at Helsinki University of Technology (HUT) [21], [22] and Brigham Young University (BYU) [23], [24] confirms the utility of the model in real environments in terms of mutual information, several types of correlations, level crossing rate (LCR), and average fade duration (AFD).

The rest of this paper is organized as follows. The singlebounce two-ring model is presented in Section II. The closedform expression for the STCC function between any two subchannels is derived in Section III. Section IV includes data processing and parameter-estimation techniques. In Section V, we compare the proposed model with the measured data from HUT and BYU, whose details are given in Appendixes B and C. Finally, concluding remarks are provided in Section VI.

Notation: $\|\cdot\|_{F}$ is reserved for the Frobenius norm, $(\cdot)^{\dagger}$ for the matrix Hermitian, $(\cdot)^{\mathrm{T}}$ for the matrix transpose, $(\cdot)^{\star}$ for the complex conjugate, $\mathbb{E}[\cdot]$ for the mathematical expectation, and $\jmath$ for $\sqrt{-1}$. $\mathbf{I}_{m}$ denotes the $m \times m$ identity matrix, and $[\mathbf{A}]_{m, n}$ is the $(m, n)$ th element of the matrix A. $t \in[m, n]$ with integers $m$ and $n$ implies that $t$ is an integer such that $m \leqslant t \leqslant n$, whereas $t \in\left[z_{1}, z_{2}\right)$ with real $z_{1}$ and $z_{2}$ denotes that $t$ is real such that $z_{1} \leqslant t<z_{2} . \min (x, y)$ and $\max (x, y)$ indicate the minimum and maximum of real $x$ and $y$, respectively. $\operatorname{vec}(\mathbf{A})$ stacks all the columns of the matrix $\mathbf{A}$ into one tall column vector.

\section{Proposed Statistical Model}

For a linear time-varying flat fading $N_{\mathrm{R}} \times N_{\mathrm{T}}$ MIMO system, the input-output relationship can be written as

$$
\mathbf{r}(t)=\sqrt{\frac{P_{\mathrm{Tx}}}{N_{\mathrm{T}}}} \mathbf{H}(t) \mathbf{s}(t)+\mathbf{e}(t)
$$

In (1) $P_{\mathrm{Tx}}$ is the total emitted power from the Tx antenna array. $[\mathbf{H}(t)]_{l, p}=h_{l p}(t), h_{l p}(t)$ denotes the complex baseband equivalent channel gain between the $p$ th transmitter and the $l$ th receiver. $\mathbf{r}(t)=\left[r_{1}(t), r_{2}(t), \ldots, r_{N_{\mathrm{R}}}(t)\right]^{\mathrm{T}}$ is the received signal vector at time $t$. $\mathbf{s}(t)=\left[s_{1}(t), s_{2}(t), \ldots, s_{N_{\mathrm{T}}}(t)\right]^{\mathrm{T}}$ is the transmitted symbol vector from $N_{\mathrm{T}}$ Tx antennas at time $t$ such that each element of $\mathbf{s}(t)$ has a unit power. $\mathbf{e}(t)=$ $\left[e_{1}(t), e_{2}(t), \ldots, e_{N_{\mathrm{R}}}(t)\right]^{\mathrm{T}}$ is the additive white Gaussian noise (AWGN) vector with the covariance matrix $\mathbf{R}=$ $\mathbb{E}\left[\mathbf{e}(t) \mathbf{e}^{\dagger}(t)\right]=P_{\text {Noise }} \mathbf{I}_{N_{\mathrm{R}}}$ [5], where $P_{\text {Noise }}$ is the average noise power at each $\mathrm{Rx}$ antenna element.

The geometry of the proposed model is shown in Fig. 1 for a $2 \times 2$ channel where scatterers that are local to the BS and MS are modeled to be distributed on two separate rings. The key difference between our model and the other two-ring model [13] is that only single-bounce rays are considered, and multiple bounces are treated as secondary effects. This avoids the problem of the double-bounce two-ring model discussed in [13], facilitates the derivation of closed-form results, and proves to be a useful approximation when compared with the measured data.

In Fig. 1, which shows the forward channel (from the BS to the MS), the MS receives single-bounce rays from the scatterer $S_{i}$ around the MS (shown by dotted lines) and the scatterer $S_{k}^{\prime}$ around the BS (shown by dash-dotted lines). $D$ is the distance between the MS and $\mathrm{BS}, R$ and $R^{\prime}$ are the radii of the scattering rings around the MS and BS, respectively, and $\alpha_{p q}$ and $\beta_{l m}$ are the directions of the subarray $\mathrm{BS}_{p}-\mathrm{BS}_{q}$ with element spacing $\delta_{p q}$ and subarray $\mathrm{MS}_{l}$-MS $m$ with element spacing $d_{l m}$, respectively. ${ }^{3}$ For the frequency flat subchannel between the antenna elements $\mathrm{BS}_{p}$ and $\mathrm{MS}_{l}, h_{l p}(t)$ denotes the time-varying complex baseband equivalent channel gain. Mathematical representation of the superposition of rays at the MS results in the following expression for the channel gain ${ }^{4}$ :

$$
\begin{array}{r}
h_{l p}(t)=\sqrt{\eta^{\prime} \Omega_{l p}} \sum_{k=1}^{N^{\prime}} \frac{g_{k}^{\prime}}{\sqrt{N^{\prime}}} \exp \left[\jmath \psi_{k}^{\prime}-\frac{\jmath 2 \pi}{\lambda}\left(\xi_{p k}^{\prime}+\xi_{k l}^{\prime}\right)\right. \\
\left.+\jmath 2 \pi f_{D} \cos \left(\varphi_{k}^{\prime}-\gamma\right) t\right] \\
+\sqrt{\eta \Omega_{l p}} \sum_{i=1}^{N} \frac{g_{i}}{\sqrt{N}} \exp \left[\jmath \psi_{i}-\frac{\jmath 2 \pi}{\lambda}\left(\xi_{p i}+\xi_{i l}\right)\right. \\
\left.+\jmath 2 \pi f_{D} \cos \left(\phi_{i}-\gamma\right) t\right]
\end{array}
$$

\footnotetext{
${ }^{3}$ Note that $\alpha_{q p}=\alpha_{p q}+\pi, p<q, \quad \beta_{m l}=\beta_{l m}+\pi, l<m, \quad \delta_{p q}=$ $\delta_{q p} \geqslant 0, \forall p, q$, and $d_{l m}=d_{m l} \geqslant 0, \forall l, m$, with $p, q, l, m$ being the positive integers.

${ }^{4}$ To save space, only non-line-of-sight (NLOS) components are considered in (2). It can be easily extended to include the LOS component by introducing the Rician $K$ factor if the number of scatters is large, in the same way as in [5].
} 


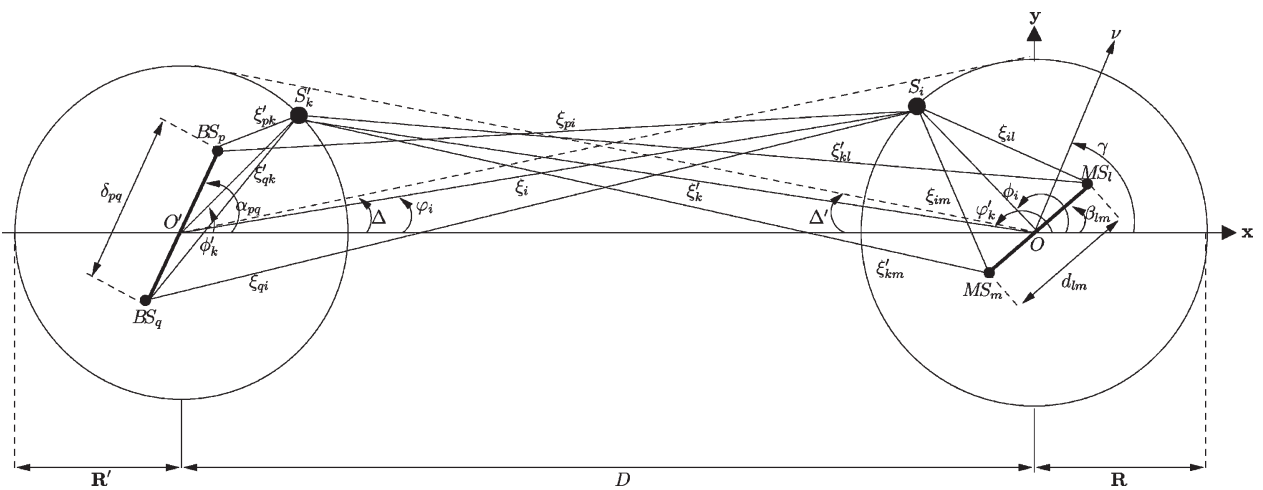

Fig. 2. Detailed version of Fig. 1.

where the first and second summations correspond to the BS and MS rings, respectively. From (2), it is clear that the angle of arrival (AoA) and the angle of departure (AoD) play the interrelation between the single-bounce two-ring model in Fig. 2 and the channel transfer function $\mathbf{H}(t)$ in (1).

In (2), $\Omega_{l p}$ is the power transmitted through the subchannel $\mathrm{BS}_{p}-\mathrm{MS}_{l}$, i.e., $\Omega_{l p}=\mathbb{E}\left[\left|h_{l p}\right|^{2}\right], \eta^{\prime}$ and $\eta$ show the respective contributions of scatterers around the BS and MS to $\Omega_{l p}$ such that $\eta^{\prime}+\eta=1$, and $N$ and $N^{\prime}$ are the numbers of scatterers around the MS and BS, respectively. The positive random variables $g_{i}$ and $g_{k}^{\prime}$ represent the amplitudes of the waves scattered by $S_{i}$ and $S_{k}^{\prime}$, which also include the antenna gains at $\phi_{i}$ and $\phi_{k}^{\prime}$, and $\psi_{i}$ and $\psi_{k}^{\prime}$ are the associated phase shifts. Furthermore, as shown in Fig. 2, $\phi_{k}^{\prime}$ and $\varphi_{i}$ are the AoDs of the waves that impinge on $S_{k}^{\prime}$ and $S_{i}$, whereas $\varphi_{k}^{\prime}$ and $\phi_{i}$ are the AoAs of the waves scattered from $S_{k}^{\prime}$ and $S_{i} . \xi_{p k}^{\prime}$ and $\xi_{q k}^{\prime}$, which are functions of $\phi_{k}^{\prime}$, are the lengths of $\mathrm{BS}_{p}-S_{k}^{\prime}$ and $\mathrm{BS}_{q}-S_{k}^{\prime}$ links, respectively, whereas $\xi_{k l}^{\prime}$ and $\xi_{k m}^{\prime}$, which are functions of $\varphi_{k}^{\prime}$, are the lengths of $S_{k}^{\prime}$ $\mathrm{MS}_{l}$ and $S_{k}^{\prime}-\mathrm{MS}_{m}$ links. Other $\xi$ 's can be easily identified in Fig. 2, and their functional relationships are given in (26a)(26h). Finally, $\lambda, f_{D}$, and $\gamma$ are the wavelength, maximum Doppler frequency, and the direction of the MS motion, respectively.

The sets $\left\{g_{i}\right\}_{i=1}^{N}$ and $\left\{g_{k}^{\prime}\right\}_{k=1}^{N^{\prime}}$ consist of independent positive random variables with finite variances, independent of $\left\{\psi_{i}\right\}_{i=1}^{N}$ and $\left\{\psi_{k}^{\prime}\right\}_{k=1}^{N^{\prime}}$. We assume that $\left\{\psi_{i}\right\}_{i=1}^{N}$ and $\left\{\psi_{k}^{\prime}\right\}_{k=1}^{N^{\prime}}$ are the independent identically distributed (i.i.d.) random variables with uniform distributions over $[0,2 \pi)$. We also set $N^{-1} \sum_{i=1}^{N} \mathbb{E}\left[g_{i}^{2}\right]=1$ and $N^{\prime-1} \sum_{k=1}^{N^{\prime}} \mathbb{E}\left[g_{k}^{\prime 2}\right]=1$, which result in the desired identity $\mathbb{E}\left[\left|h_{l p}(t)\right|^{2}\right]=\Omega_{l p}$. The second moments of $g_{i}, \forall i$, and $g_{k}^{\prime}, \forall k$ will be discussed in Section III. According to Fig. 2, $\varphi_{i}$ is a function of $\phi_{i}$, and $\varphi_{k}^{\prime}$ is a function of $\phi_{k}^{\prime}$; therefore, only $\phi_{k}^{\prime}$ and $\phi_{i}$ are the independent angular variables. In what follows, we call $\phi_{k}^{\prime}$ the AoD and $\phi_{i}$ the AoA.

\section{Mimo Spatio-Temporal Cross CoRrelation}

Based on the independent properties of $g_{i}$ 's, $g_{k}^{\prime}$ 's, $\psi_{i}$ 's, and $\psi_{k}^{\prime}$ 's, the normalized STCC between the two subchannel gains $h_{l p}(t)$ and $h_{m q}(t)$, which are defined as $\rho_{l p, m q}(\tau)=\mathbb{E}\left[h_{l p}(t+\right.$ $\left.\tau) h_{m q}^{\star}(t)\right] / \sqrt{\Omega_{l p} \Omega_{m q}}, 5$ can be asymptotically written as

$$
\begin{aligned}
\rho_{l p, m q}(\tau)= & \lim _{N^{\prime} \rightarrow \infty} \frac{(1-\eta)}{N^{\prime}} \sum_{k=1}^{N^{\prime}} \mathbb{E}\left[g_{k}^{\prime 2}\right] \\
& \times \exp \left[-\jmath \frac{2 \pi}{\lambda}\left(\xi_{p k}^{\prime}-\xi_{q k}^{\prime}+\xi_{k l}^{\prime}-\xi_{k m}^{\prime}\right)\right. \\
& \left.\quad+\jmath 2 \pi f_{D} \cos \left(\varphi_{k}^{\prime}-\gamma\right) \tau\right]+\lim _{N \rightarrow \infty} \frac{\eta}{N} \\
\times & \quad \sum_{i=1}^{N} \mathbb{E}\left[g_{i}^{2}\right] \exp \left[-\jmath \frac{2 \pi}{\lambda}\left(\xi_{p i}-\xi_{q i}+\xi_{i l}-\xi_{i m}\right)\right. \\
& \left.\quad+\jmath 2 \pi f_{D} \cos \left(\phi_{i}-\gamma\right) \tau\right] .
\end{aligned}
$$

For large $N^{\prime}$ and $N$, the small amounts of power received from $S_{k}^{\prime}$ and $S_{i}$ are proportional to $N^{\prime-1} \mathbb{E}\left[g_{k}^{\prime 2}\right]$ and $N^{-1} \mathbb{E}\left[g_{i}^{2}\right]$, respectively. They are also equal to the infinitesimal powers through the differential angles $d \phi^{\prime}$ and $d \phi$ with probabilities $f_{\mathrm{BS}}\left(\phi_{k}^{\prime}\right) d \phi^{\prime}$ and $f_{\mathrm{MS}}\left(\phi_{i}\right) d \phi$, respectively. This implies that $N^{\prime-1} \mathbb{E}\left[g_{k}^{\prime 2}\right]=f_{\mathrm{BS}}\left(\phi_{k}^{\prime}\right) d \phi^{\prime}$ and $N^{-1} \mathbb{E}\left[g_{i}^{2}\right]=f_{\mathrm{MS}}\left(\phi_{i}\right) d \phi$, where $f_{\mathrm{BS}}(\cdot)$ is the probability density functions (pdf) of the AoD, and $f_{\mathrm{MS}}(\cdot)$ is the pdf of the AoA. To simplify the notation, we define $x=\phi_{k}^{\prime}, y=\phi_{i}, v=\varphi_{k}^{\prime}$, and $w=\varphi_{i}$. Therefore, (3) can be reduced to the following integral form:

$$
\begin{array}{r}
\rho_{l p, m q}(\tau)=(1-\eta) \int_{-\pi}^{\pi} \exp \left[-\frac{\jmath 2 \pi}{\lambda}\left(\xi_{p x}^{\prime}-\xi_{q x}^{\prime}+\xi_{x l}^{\prime}-\xi_{x m}^{\prime}\right)\right. \\
\left.\quad+\jmath 2 \pi f_{D} \cos (v-\gamma) \tau\right] f_{\mathrm{BS}}(x) d x \\
+\eta \int_{-\pi}^{\pi} \exp \left[-\frac{j 2 \pi}{\lambda}\left(\xi_{p y}-\xi_{q y}+\xi_{y l}-\xi_{y m}\right)\right. \\
\left.+\jmath 2 \pi f_{D} \cos (y-\gamma) \tau\right] f_{\mathrm{MS}}(y) d y
\end{array}
$$

where $\xi^{\prime}$ 's depend on $\phi_{k}^{\prime}$, and $\xi$ 's depend on $\phi_{i}$, according to Fig. 2.

\footnotetext{
${ }^{5}$ This definition is the same as the one in [5] if $\tau$ is replaced by $-\tau$.
} 
For any given $f_{\mathrm{BS}}(\cdot)$ and $f_{\mathrm{MS}}(\cdot)$, (4) can be calculated numerically using the trigonometric function relationships given in (26a)-(26h), (30a), and (30b). Note that (4) includes two parts; the first one corresponds to the STCC contributed by the scattering ring around the BS, and the second is from the scattering ring around the MS.

In order to further simplify (4), similarly to [5], the assumptions of $D \gg \max \left(R^{\prime}, R\right), R^{\prime} \gg \delta_{p q}$ and $R \gg d_{l m}$ are made. ${ }^{6}$ With these assumptions and the identities given in (26a)-(26h), (4) can be approximated by

$$
\begin{gathered}
\rho_{l p, m q}(\tau) \approx(1-\eta) \int_{-\pi}^{\pi} \exp \left\{j 2 \pi \left[\delta_{p q} \cos \left(\alpha_{p q}-x\right)\right.\right. \\
\left.+d_{l m} \cos \left(v-\beta_{l m}\right)\right] / \lambda \\
\left.+\jmath 2 \pi f_{D} \cos (v-\gamma) \tau\right\} f_{\mathrm{BS}}(x) d x \\
+\eta \int_{-\pi}^{\pi} \exp \left\{\jmath 2 \pi \left[\delta_{p q} \cos \left(\alpha_{p q}-w\right)\right.\right. \\
\left.+d_{l m} \cos \left(y-\beta_{l m}\right)\right] / \lambda \\
\left.+\jmath 2 \pi f_{D} \cos (y-\gamma) \tau\right\} f_{\mathrm{MS}}(y) d y .
\end{gathered}
$$

The details of the derivation are given in Appendix A.

In this paper, we consider the empirically verified von Mises angular pdf [25] for both the AoD and AoA. ${ }^{7}$ They are given by

$$
\begin{array}{ll}
f_{\mathrm{BS}}(x)=\frac{\exp \left[\kappa^{\prime} \cos \left(x-\mu^{\prime}\right)\right]}{2 \pi I_{0}\left(\kappa^{\prime}\right)}, & x \in[0,2 \pi) \\
f_{\mathrm{MS}}(y)=\frac{\exp [\kappa \cos (y-\mu)]}{2 \pi I_{0}(\kappa)}, & y \in[0,2 \pi) .
\end{array}
$$

In (6a) and (6b), $I_{k}(z)=(1 / \pi) \int_{0}^{\pi} e^{z \cos \theta} \cos (k \theta) d \theta$ is the $k$ thorder modified Bessel function of the first kind, $\mu^{\prime}$ and $\mu$ account for the mean AoD and mean AoA, respectively, and $\kappa^{\prime}$ and $\kappa(\geqslant 0)$ control the angular spreads of the AoD and AoA, respectively. If $\kappa=\kappa^{\prime}=0$, both pdfs in (6) simplify to $1 / 2 \pi$, which represents isotropic scattering. As shown in Appendix A, the mathematical form of the von Mises pdf is convenient for the analytic calculations and derivations of closed-form expressions.

\footnotetext{
${ }^{6}$ These assumptions can be justified in many propagation scenarios of practical interest. In certain picocell propagation environments, however, $D \gg$ $\max \left(R^{\prime}, R\right)$ may not strictly hold. This may result in some discrepancies between the model and measured data in some cases, as discussed in Section V-E.

${ }^{7}$ In this paper, no preassumption is made about the antenna patterns. In fact, $g_{k}^{\prime}$ 's and $g_{i}$ 's include the Tx and Rx antenna gains, and the angular pdfs in (6a) and (6b) include the effect of antenna patterns.
}

Based on (30a), (30b), (33), and the assumption of $D \gg$ $\max \left(R^{\prime}, R\right)$, the following closed-form expression for the STCC is derived in Appendix A:

$$
\begin{aligned}
& \rho_{l p, m q}(\tau) \approx(1-\eta) \frac{\exp \left[-\jmath\left(b_{l m} \cos \beta_{l m}-a \cos \gamma\right)\right]}{I_{0}\left(\kappa^{\prime}\right)} \\
& \times I_{0}\left(\left\{\kappa^{\prime 2}-a^{2} \Delta^{\prime 2} \sin ^{2} \gamma-b_{l m}^{2} \Delta^{\prime 2} \sin ^{2} \beta_{l m}\right.\right. \\
& \quad-c_{p q}^{2}-2 b_{l m} c_{p q} \Delta^{\prime} \sin \alpha_{p q} \sin \beta_{l m} \\
&+2 a \Delta^{\prime} \sin \gamma\left(c_{p q} \sin \alpha_{p q}+b_{l m} \Delta^{\prime} \sin \beta_{l m}\right) \\
& \quad-\jmath 2 \kappa^{\prime}\left[a \Delta^{\prime} \sin \mu^{\prime} \sin \gamma-b_{l m} \Delta^{\prime} \sin \beta_{l m}\right. \\
&\left.\left.\left.\times \sin \mu^{\prime}-c_{p q} \cos \left(\alpha_{p q}-\mu^{\prime}\right)\right]\right\}^{\frac{1}{2}}\right) \\
&+\eta \frac{\exp \left(\jmath c_{p q} \cos \alpha_{p q}\right)}{I_{0}(\kappa)} \\
& \times I_{0}\left(\left\{\kappa^{2}-a^{2}-b_{l m}^{2}-c_{p q}^{2} \Delta^{2} \sin { }^{2} \alpha_{p q}\right.\right. \\
& \quad+2 c_{p q} \Delta \sin \alpha_{p q} \times\left(a \sin \gamma-b_{l m} \sin \beta_{l m}\right) \\
&+2 a b_{l m} \cos \left(\beta_{l m}-\gamma\right)-\jmath^{2 \kappa} \\
& \times\left[a \cos (\mu-\gamma)-b_{l m} \cos \left(\beta_{l m}-\mu\right)\right. \\
&\left.\left.\left.\quad-c_{p q} \Delta \sin \alpha_{p q} \sin \mu\right]\right\}^{\frac{1}{2}}\right) .
\end{aligned}
$$

Here, we have $a=-2 \pi f_{D} \tau, b_{l m}=2 \pi d_{l m} / \lambda$, and $c_{p q}=$ $2 \pi \delta_{p q} / \lambda$. Furthermore, $2 \Delta^{\prime}$ is the maximum angle spread at the MS, which is determined by the scattering ring around the BS. Similarly, $2 \Delta$ is the maximum angle spread at the BS, which is dictated by the scattering ring around the MS. Note that (7) is a closed-form STCC function between any two subchannels of a MIMO system with arbitrary array configurations, i.e., Tx and Rx arrays do not have to be linear. In what follows, we show that many existing correlation models can be considered as special cases of (7).

1) If there is no scatterer around the BS as in a macrocell $(\eta=1)$, the first half of (7) disappears, and the remaining part is the same as (12) in [5], when $\tau$ is changed to ${ }^{5}$ $-\tau$. It implies that (7) includes the model of [5] and, subsequently, other models listed in [5] as special cases.

2) With $l=m$ and $p=q$, the temporal autocorrelation of the subchannel $h_{l p}(t)$ can be derived from (7) as

$$
\begin{array}{r}
\rho(\tau)=\eta \frac{I_{0}\left(\sqrt{\kappa^{2}-a^{2}-\jmath 2 \kappa a \cos (\mu-\gamma)}\right)}{I_{0}(\kappa)}+(1-\eta) \\
\times \frac{I_{0}\left(\sqrt{\kappa^{\prime 2}-a^{2} \Delta^{\prime 2} \sin ^{2} \gamma-\jmath 2 \kappa^{\prime} a \Delta^{\prime} \sin \mu^{\prime} \sin \gamma}\right)}{e^{-\jmath a \cos \gamma} I_{0}\left(\kappa^{\prime}\right)}
\end{array}
$$

where $\rho(\tau)=\rho_{l p, l p}(\tau), \forall l, p$. If $\eta=1,(8)$ reduces to the model of [25].

a) With $\eta=1$ and $\kappa=0$ (isotropic scattering around the MS), (8) simplifies to the well-known Clarke's temporal correlation model, i.e., $J_{0}\left(2 \pi f_{D} \tau\right)$ [26], where $J_{0}(\cdot)$ is the Bessel function of the first kind of order zero. 
3) When the MS does not move, for example, in indoor environments, one gets $f_{D}=0$. This reduces (7) to the following spatial correlation between $h_{l p}$ and $h_{m q}$ :

$$
\begin{aligned}
\rho_{l p, m q}=(1- & \eta) \frac{e^{-\jmath b_{l m} \cos \beta_{l m}}}{I_{0}\left(\kappa^{\prime}\right)} \\
\times & I_{0}\left(\left\{\kappa^{\prime 2}-b_{l m}^{2} \Delta^{\prime 2} \sin ^{2} \beta_{l m}-c_{p q}^{2}\right.\right. \\
& \quad-2 b_{l m} c_{p q} \Delta^{\prime} \sin \alpha_{p q} \sin \beta_{l m}+\jmath 2 \kappa^{\prime} \\
& \left.\left.\quad \times\left[b_{l m} \Delta^{\prime} \sin \beta_{l m} \sin \mu^{\prime}+c_{p q} \cos \left(\alpha_{p q}-\mu^{\prime}\right)\right]\right\}^{\frac{1}{2}}\right) \\
+ & \eta \frac{e^{\jmath c_{p q} \cos \alpha_{p q}}}{I_{0}(\kappa)} \\
\times & I_{0}\left(\left\{\kappa^{2}-b_{l m}^{2}-c_{p q}^{2} \Delta^{2} \sin ^{2} \alpha_{p q}\right.\right. \\
& \quad-2 b_{l m} c_{p q} \Delta \sin \alpha_{p q} \sin \beta_{l m}+\jmath 2 \kappa \\
& \left.\left.\times\left[b_{l m} \cos \left(\beta_{l m}-\mu\right)+c_{p q} \Delta \sin \alpha_{p q} \sin \mu\right]\right\}^{\frac{1}{2}}\right) .
\end{aligned}
$$

a) With isotropic scattering around both the BS and MS $\left(\kappa^{\prime}=\kappa=0\right)$, and parallel linear arrays $\left(\alpha_{p q}=\beta_{l m}=\right.$ $\pi / 2, \forall p, q, l, m$ ), the spatial correlation in (9) further simplifies to

$$
\rho_{l p, m q}=(1-\eta) J_{0}\left(b_{l m} \Delta^{\prime}+c_{p q}\right)+\eta J_{0}\left(b_{l m}+c_{p q} \Delta\right) .
$$

With $p=q$ and $\eta=1$, (10) reduces to $\rho_{l p, m p}=$ $J_{0}\left(2 \pi d_{l m} / \lambda\right)$. It is the spatial correlation at the MS in a macrocell [26]. On the other hand, with $l=m$ and $\eta=1$, (10) simplifies to $\rho_{l p, l q}=J_{0}\left(\Delta 2 \pi \delta_{p q} / \lambda\right)$. It is the spatial correlation at the BS in a macrocell [27].

\section{Data Processing and Parameter Estimation}

This section explains how the raw data are processed and how the parameters of the proposed model are estimated.

\section{A. Normalization}

First, it is necessary to do some power normalization. In [28], the normalization is done according to $\widetilde{\mathbf{H}}(t)=$ $\sqrt{N_{\mathrm{T}} N_{\mathrm{R}}} \mathbf{H}(t) /\|\mathbf{H}(t)\|_{F}$ for each $t, t \in[1, T]$, where $\mathbf{H}(t)$ is a snapshot of the channel matrix at time instant $t, T$ is the total number of snapshots, and $\|\mathbf{H}(t)\|_{F}=\sqrt{\sum_{l=1}^{N_{\mathrm{R}}} \sum_{p=1}^{N_{\mathrm{T}}}\left|h_{l p}(t)\right|^{2}}$. Note that $\mathbf{H}(t)$ denotes $\mathbf{H}\left(t T_{\mathrm{s}}\right)$ and $T_{\mathrm{s}}$ is the sampling period. To simplify notations, $T_{\mathrm{s}}$ is dropped in this section. In [29], normalization is intended to guarantee the so-called unit singleinput single-output gain, i.e., $T^{-1} \sum_{t=1}^{T}\|\widetilde{\mathbf{H}}(t)\|_{F}^{2}=N_{\mathrm{T}} N_{\mathrm{R}}$; therefore, the normalization is done according to

$$
\widetilde{\mathbf{H}}(t)=\sqrt{\frac{N_{\mathrm{R}} N_{\mathrm{T}} T}{\sum_{t=1}^{T}\|\mathbf{H}(t)\|_{F}^{2}}} \mathbf{H}(t), \quad t \in[1, T] .
$$

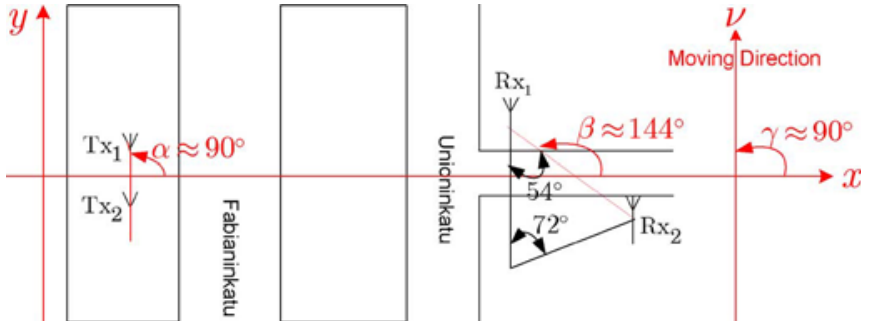

Fig. 3. Estimating $\alpha, \beta$, and $\gamma$ by a comparison of Figs. 12 and 13 with Fig. 1.

According to our correlation model in (7), each individual subchannel has unit power. On the other hand, the aforementioned two normalization methods do not result in unit power for each subchannel. Hence, in this paper, normalization is performed such that each subchannel has unit variance and zero mean, i.e.,

$$
\tilde{h}_{l p}(t)=\frac{h_{l p}(t)-\widehat{m}_{l p}}{\widehat{\sigma}_{l p}}, \quad \forall l, p, t
$$

where $\widehat{m}_{l p}$ and $\widehat{\sigma}_{l p}^{2}$ are the estimated mean and variance of the $\mathrm{BS}_{p}-\mathrm{MS}_{l}$ subchannel. They are calculated from $\left\{h_{l p}(t)\right\}_{t=1}^{T}$ according to $\widehat{m}_{l p}=T^{-1} \sum_{t=1}^{T} h_{l p}(t)$ and $\widehat{\sigma}_{l p}^{2}=$ $T^{-1} \sum_{t=1}^{T}\left|h_{l p}(t)-\widehat{m}_{l p}\right|^{2}$.

\section{B. Parameter Estimation}

First, we need to determine $\alpha$ and $\beta$, which are the directions of BS and MS arrays, respectively. ${ }^{8}$ The direction of the MS motion $\gamma$ and the Doppler drift $f_{D}$ in the HUT data needs to be obtained as well, whereas the fixed MS in the BYU data does not need $\gamma$ and $f_{D}$.

1) For the HUT data, a comparison of Figs. 12 and 13 with Fig. 1 gives $\alpha=\pi / 2, \beta=4 \pi / 5$, and $\gamma=\pi / 2$. To obtain these angles, first, $x$ - and $y$-axes were chosen in the horizontal and vertical directions, respectively, similarly to Fig. 1, and were redrawn on Fig. 12. This results in Fig. 3. A comparison of Fig. 3 with Fig. 1 reveals that the BS array is parallel to the $y$-axis; therefore, $\alpha=\pi / 2 .{ }^{9} \beta$ can be determined similarly as $144^{\circ}=180^{\circ}-(90-54)^{\circ}$ based on the $54^{\circ}$ angle shown in Fig. 3. A further comparison of Fig. 3 with Fig. 1 shows that the mobile is moving parallel to the $y$-axis in the positive direction. Therefore, $\gamma=\pi / 2$. The data are taken over a $47-\mathrm{m}$ route in Unioninkatu Street, at the intersection with Rauhankatu Street, as shown in Fig. 12. $f_{D}=2.872 \mathrm{~Hz}$, which is derived in Appendix B. Note that only a small section

\footnotetext{
${ }^{8}$ In BYU measurements, both the Tx and Rx arrays are linear, and the HUT data used in this paper is a $2 \times 2$ channel taken from a large MIMO system. Therefore, in both measurement setups, we have $\alpha_{p q}=\alpha, p<q$ and $\beta_{l m}=$ $\beta, l<m$.

${ }^{9}$ Note that based on our convention, this $\alpha$ is indeed $\alpha_{12}$ (refer to footnote 8). As described in footnote 3 , one cannot choose $3 \pi / 2$ for $\alpha_{12}$. Therefore, based on our convention, $\alpha=\pi / 2$ is the only choice in Fig. 3 .
} 
( $\approx 20 \mathrm{~m})$ around Rauhankatu Street is used. Therefore, the estimated $\alpha, \beta$, and $\gamma$ are a fair approximation for all locations since $20 \mathrm{~m}$ is small compared to the distance of $150 \mathrm{~m}$.

2) Similarly, $\alpha=168^{\circ}$ and $\beta=78^{\circ}$ are obtained for the BYU data according to Figs. 1 and 14.

To estimate the rest of the parameters $\boldsymbol{\Lambda}=$ $\left(\Delta, \Delta^{\prime}, \kappa, \kappa^{\prime}, \mu, \mu^{\prime}, \eta\right)$ of the model, a nonlinear least-square correlation fitting approach is used, via a numerical search over the parameter space. This is explained next.

For the BYU data, $\Lambda$ is estimated ${ }^{10}$ by fitting the spatial MIMO correlation matrix $\mathbf{R}(\boldsymbol{\Lambda})$, whose elements are given by (9), to the estimated spatial correlation matrix $\widehat{\mathbf{R}}$

$$
\widehat{\boldsymbol{\Lambda}}=\arg \min _{\boldsymbol{\Lambda}}\|\widehat{\mathbf{R}}-\mathbf{R}(\boldsymbol{\Lambda})\|_{F}^{2}
$$

$\widehat{\mathbf{R}}=T^{-1} \sum_{t=1}^{T} \mathbf{h}(t) \mathbf{h}^{\dagger}(t)$ with $\mathbf{h}(t)=\operatorname{vec}(\mathbf{H}(t)) . \mathbf{R}(\boldsymbol{\Lambda})=$ $\mathbb{E}\left[\mathbf{h}(t) \mathbf{h}^{\dagger}(t)\right]$ such that $[\mathbf{R}(\boldsymbol{\Lambda})]_{l+N_{\mathrm{R}}(p-1), m+N_{\mathrm{R}}(q-1)}=\rho_{l p, m q}$, $l, m \in\left[1, N_{\mathrm{R}}\right], p, q \in\left[1, N_{\mathrm{T}}\right]$. Note that the expectation is taken over AoA and AoD, as shown in (4), and $\rho_{l p, m q}$ is given by (9). Furthermore, $\mathbf{R}(\boldsymbol{\Lambda})$ is a $N_{\mathrm{T}} N_{\mathrm{R}} \times N_{\mathrm{T}} N_{\mathrm{R}}$ Hermitian block Toeplitz with Toeplitz blocks (H-BTTB) matrix. It has $N_{\mathrm{T}} \times N_{\mathrm{T}}$ blocks, and each block is an $N_{\mathrm{R}} \times N_{\mathrm{R}}$ square matrix. A matrix is BTTB if its $(i, j)$ th block is a function of $(i-j)$, and the $(i, j)$ th block itself is a Toeplitz matrix. More discussion on BTTB matrices can be found in [31]. The Hermitian property is due to the Hermitian symmetry of the STCC $\rho_{l p, m q}(\tau)$ around $\tau=0$. The BTTB structure comes from the fact that $\rho_{l p, m q}(\tau)=\rho_{(l+i)(p+j),(m+i)(q+j)}(\tau), \forall i, j$. By taking advantage of the H-BTTB structure of $\mathbf{R}(\boldsymbol{\Lambda})$, the numerical search in (13) can be performed much faster, particularly for large $N_{\mathrm{R}}$ and $N_{\mathrm{T}}$.

Similarly, for the HUT data, $\Lambda$ is estimated by fitting the spatio-temporal MIMO correlation matrix $\mathbf{R}(\boldsymbol{\Lambda}, k)$, whose elements are determined by (7), to the estimated spatio-temporal correlation matrix $\widehat{\mathbf{R}}(k)$

$$
\widehat{\boldsymbol{\Lambda}}=\arg \min _{\boldsymbol{\Lambda}} \sum_{k=0}^{\tau_{\max }}\|\widehat{\mathbf{R}}(k)-\mathbf{R}(\boldsymbol{\Lambda}, k)\|_{F}^{2}
$$

where $\tau_{\max }$ is set to 4 in the numerical search. ${ }^{11} \widehat{\mathbf{R}}(k)=$ $T^{-1} \sum_{t=1}^{T} \mathbf{h}(t+k) \mathbf{h}^{\dagger}(t), k \in\left[0, \tau_{\max }\right] . \mathbf{R}(\boldsymbol{\Lambda}, k)=\mathbb{E}[\mathbf{h}(t+$ $\left.k) \mathbf{h}^{\dagger}(t)\right]$ such that $[\mathbf{R}(\boldsymbol{\Lambda}, k)]_{l+N_{\mathrm{R}}(p-1), m+N_{\mathrm{R}}(q-1)}=\rho_{l p, m q}(k)$, $l, m \in\left[1, N_{\mathrm{R}}\right]$, and $p, q \in\left[1, N_{\mathrm{T}}\right]$. Note that $\rho_{l p, m q}(k)$ denotes $\rho_{l p, m q}\left(k T_{\mathrm{S}}\right)$ given by (7). In addition, it is clearly seen that $\mathbf{R}(\boldsymbol{\Lambda}, 0)$ is an H-BTTB matrix, whereas for $k \neq 0, \mathbf{R}(\boldsymbol{\Lambda}, k)$ is a BTTB matrix. In a similar manner to (13), the parameter search in (14) can be sped up using the BTTB structure of $\mathbf{R}(\boldsymbol{\Lambda}, k)$.

It is clear from (9) that if $\mu$ and $\mu^{\prime}$ are the outcomes of the search in (13), $2 \beta_{l m}-\widehat{\mu}$ and $2 \alpha_{p q}-\widehat{\mu}^{\prime}$ are the valid parameters

\footnotetext{
${ }^{10}$ The function in (13) is very complex since it depends on the seven free variables. The same observation applies to (14). Finding the optimal estimates is not the focus of this paper. This is a separate issue which is recently studied in [30].

${ }^{11}$ According to Appendix B, $\tau_{\max }=4$ corresponds to $1 / f_{D}$ sec.
}

TABLE I

Estimated PARAMETER SETS FOR THE HUT AND BYU DATA

\begin{tabular}{|c||c|c|c|c|c|c|c|}
\hline & $\widehat{\Delta}$ & $\widehat{\Delta}^{\prime}$ & $\widehat{\kappa}$ & $\widehat{\kappa}^{\prime}$ & $\widehat{\mu}$ & $\widehat{\mu}^{\prime}$ & $\widehat{\eta}$ \\
\hline$\widehat{\Lambda}_{\mathrm{HUT}}$ & $\frac{\pi}{6}$ & $\frac{\pi}{4}$ & 17 & 2 & $\frac{9 \pi}{8}$ & $\frac{15 \pi}{8}$ & 0.7 \\
\hline$\widehat{\Lambda}_{\mathrm{BYU}}$ & $\frac{\pi}{3}$ & $\frac{\pi}{6}$ & 0 & 0.5 & 0 & $\frac{5 \pi}{8}$ & 0.2 \\
\hline
\end{tabular}

as well and result in the same numerical values for (9). On the other hand, if $\mu^{\prime}$ is the search result of (14), $2 \alpha_{p q}-\widehat{\mu}^{\prime}$ gives the same numerical value for (7) as well. All these angular ambiguities should be considered with the real propagation environments when looking at the search results of (13) and (14). Moreover, for the HUT data, the nonuniform antenna patterns [22, Fig. 2] were also considered when searching for parameters. ${ }^{7}$ Finally, the estimated parameter sets for the HUT and BYU data are presented in Table I.

\section{Comparison of the Proposed Model With COLlected DATA}

This section presents the comparison of the proposed model with collected data in terms of various aspects of fading channels such as the statistical distribution of amplitude, phase, in-phase/quadrature components, spatial, temporal, and spatio-temporal correlations, LCR, AFD, and MIMO mutual information.

\section{A. Statistical Distribution of the Data}

When deriving the compact STCC expression in (7), it has been assumed that the numbers of local scatterers around both the BS and MS are large enough. This translates into complex Gaussian distribution for each subchannel $h_{l p}(t)$ according to the central limit theorem [32]. To verify this, the data are normalized according to (12) such that each subchannel has zero mean with unit variance. As shown in Fig. 4, the real and imaginary parts of $h_{11}(t)$ of the HUT data are Gaussian. Furthermore, the empirical cumulative distribution functions (CDFs) of the amplitude and phase are Rayleigh and uniform, respectively. The same types of plots are observed for other HUT subchannels, as well as the BYU data sets, as reported in [33, p. 162] and [34, Fig. 5].

\section{B. Spatial Correlations}

Here, we consider four different types of spatial correlations, i.e., parallel, crossing, transmit, and receive correlations, which are defined by

$$
\begin{aligned}
\rho_{\text {parallel }} & \triangleq \mathbb{E}\left[h_{l p}(t) h_{(l+u)(p+u)}^{\star}(t)\right] \\
\rho_{\text {crossing }} & \triangleq \mathbb{E}\left[h_{l p}(t) h_{p l}^{\star}(t)\right] \\
\rho_{\mathrm{Tx}} & \triangleq \mathbb{E}\left[h_{l p}(t) h_{l q}^{\star}(t)\right] \\
\rho_{\mathrm{Rx}} & \triangleq \mathbb{E}\left[h_{l p}(t) h_{m p}^{\star}(t)\right] .
\end{aligned}
$$




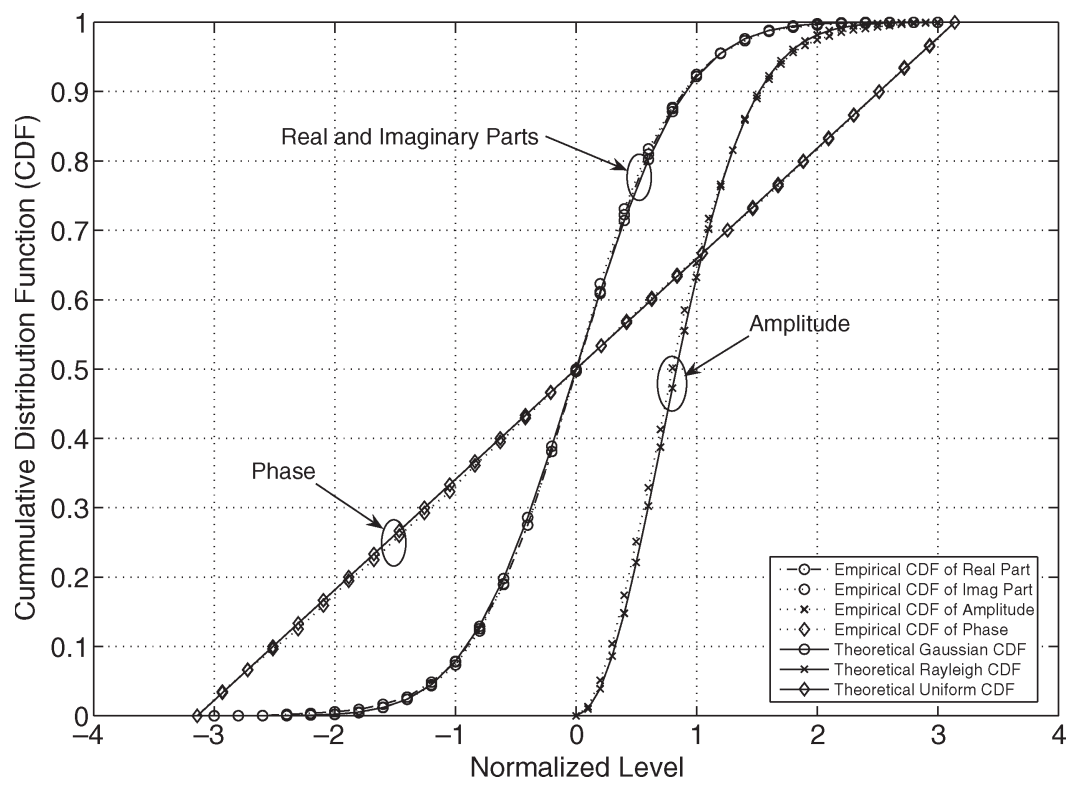

Fig. 4. Empirical and theoretical distributions of $h_{11}(t)$ in the HUT data.

In a $2 \times 2$ channel, it can be shown that

$$
\begin{aligned}
\rho_{\text {parallel }} & \triangleq \mathbb{E}\left[h_{11}(t) h_{22}^{\star}(t)\right]=\rho_{11,22} \\
\rho_{\text {crossing }} & \triangleq \mathbb{E}\left[h_{12}(t) h_{2 l}^{\star}(t)\right]=\rho_{12,21} \\
\rho_{\mathrm{Tx}} & \triangleq \mathbb{E}\left[h_{11}(t) h_{12}^{\star}(t)\right] \\
& =\mathbb{E}\left[h_{21}(t) h_{22}^{\star}(t)\right]=\rho_{11,12}=\rho_{21,22} \\
\rho_{\mathrm{Rx}} & \triangleq \mathbb{E}\left[h_{11}(t) h_{21}^{\star}(t)\right] \\
& =\mathbb{E}\left[h_{12}(t) h_{22}^{\star}(t)\right]=\rho_{11,21}=\rho_{12,22} .
\end{aligned}
$$

For the HUT data, the model correlations are obtained as $\left(\rho_{\text {parallel }}, \rho_{\text {crossing }}, \rho_{\mathrm{Tx}}, \rho_{\mathrm{Rx}}\right)=(0.01,0.2,0.5,0.02)$ by plugging $\widehat{\Lambda}_{\text {HUT }}$ from Table I into (9), whereas the empirical correlations are $(0.04,0.1,0.6,0.08)$ by processing the normalized data. For the BYU data, the same type of comparison for the aforementioned four correlations in terms of element spacing is shown in Fig. 5. The utility of the model is revealed by the close agreement between the model and the empirical spatial correlations.

\section{Spatio-Temporal Cross Correlations}

Fig. 6 shows the empirical autocorrelation of the subchannel $h_{11}(t)$ for the HUT data, together with the autocorrelation of the model obtained by plugging $\widehat{\Lambda}_{\mathrm{HUT}}$ of Table I into (8). The STCCs of the HUT data between $h_{11}(t)$ and $h_{12}(t)$ and between $h_{12}(t)$ and $h_{11}(t)$, respectively, are shown in Figs. 7 and 8 , where the cross correlation of the model is plotted by substituting $\widehat{\Lambda}_{\text {HUT }}$ of Table I into (7). The difference between the model cross correlations in Figs. 7 and 8 is due to $\alpha_{21}=$ $\alpha_{12}+\pi$, as explained in footnote 3 . Clearly, the model provides a close fit to the empirical spatio-temporal correlations.

\section{Level Crossing Rate and Average Fade Duration}

LCR and AFD of the signal envelope are two important temporal statistical features which provide useful information on the dynamic behavior of time-varying fading channels. To calculate the LCR and AFD of a subchannel, one needs its temporal autocorrelation. This is given in (8). With $\gamma=\pi / 2$ in the HUT measurement campaign, (8) reduces to

$$
\begin{array}{r}
\rho(\tau)=(1-\eta) \frac{I_{0}\left(\sqrt{\kappa^{\prime 2}-a^{2} \Delta^{\prime 2}-\jmath 2 \kappa^{\prime} a \Delta^{\prime} \sin \mu^{\prime}}\right)}{I_{0}\left(\kappa^{\prime}\right)} \\
+\eta \frac{I_{0}\left(\sqrt{\kappa^{2}-a^{2}-\jmath 2 \kappa a \sin \mu}\right)}{I_{0}(\kappa)} .
\end{array}
$$

The $k$ th spectral moment $\mathcal{B}_{k}$ is defined by [35]

$$
\mathcal{B}_{k}=\left.\frac{d^{k} \rho(\tau)}{j^{k} d \tau^{k}}\right|_{\tau=0}
$$

which is also required for calculating LCR and AFD. From (20) and (21), we obtain

$$
\begin{aligned}
& \mathcal{B}_{0}=1 \\
& \mathcal{B}_{1}=2 \pi f_{D}\left[\frac{(1-\eta) I_{1}\left(\kappa^{\prime}\right) \Delta^{\prime} \sin \mu^{\prime}}{I_{0}\left(\kappa^{\prime}\right)}+\frac{\eta I_{1}(\kappa) \sin \mu}{I_{0}(\kappa)}\right] \\
& \mathcal{B}_{2}=4 \pi^{2} f_{D}^{2}\left\{\eta\left[\sin ^{2} \mu+\frac{I_{1}(\kappa) \cos (2 \mu)}{\kappa I_{0}(\kappa)}\right]\right. \\
& \left.+(1-\eta) \Delta^{\prime 2}\left[\sin ^{2} \mu^{\prime}+\frac{I_{1}\left(\kappa^{\prime}\right) \cos \left(2 \mu^{\prime}\right)}{\kappa^{\prime} I_{0}\left(\kappa^{\prime}\right)}\right]\right\} .
\end{aligned}
$$



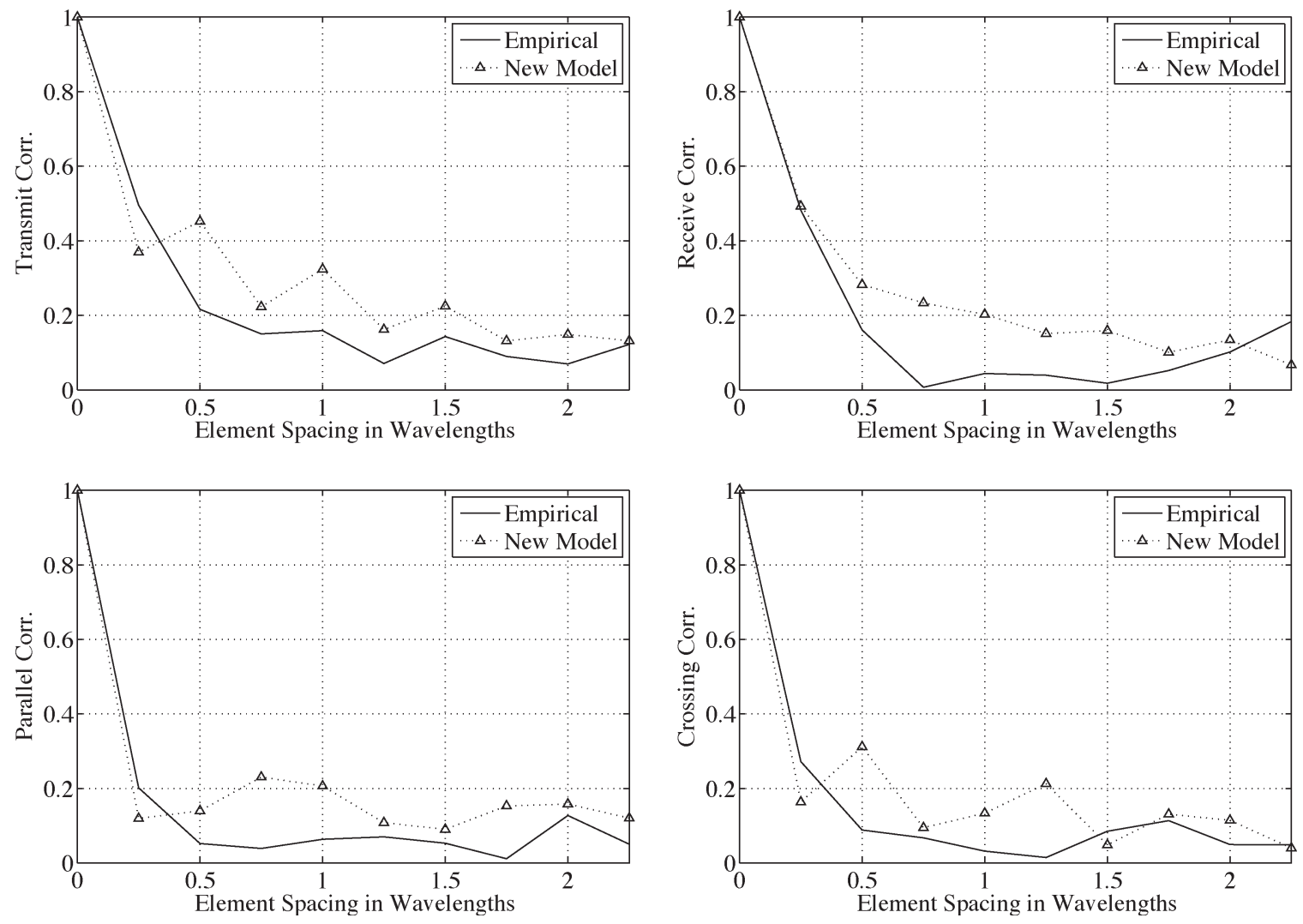

Fig. 5. Comparison of four different types of spatial correlations of the new MIMO model with the corresponding empirical correlations of the BYU data collected on November 7, 2000.

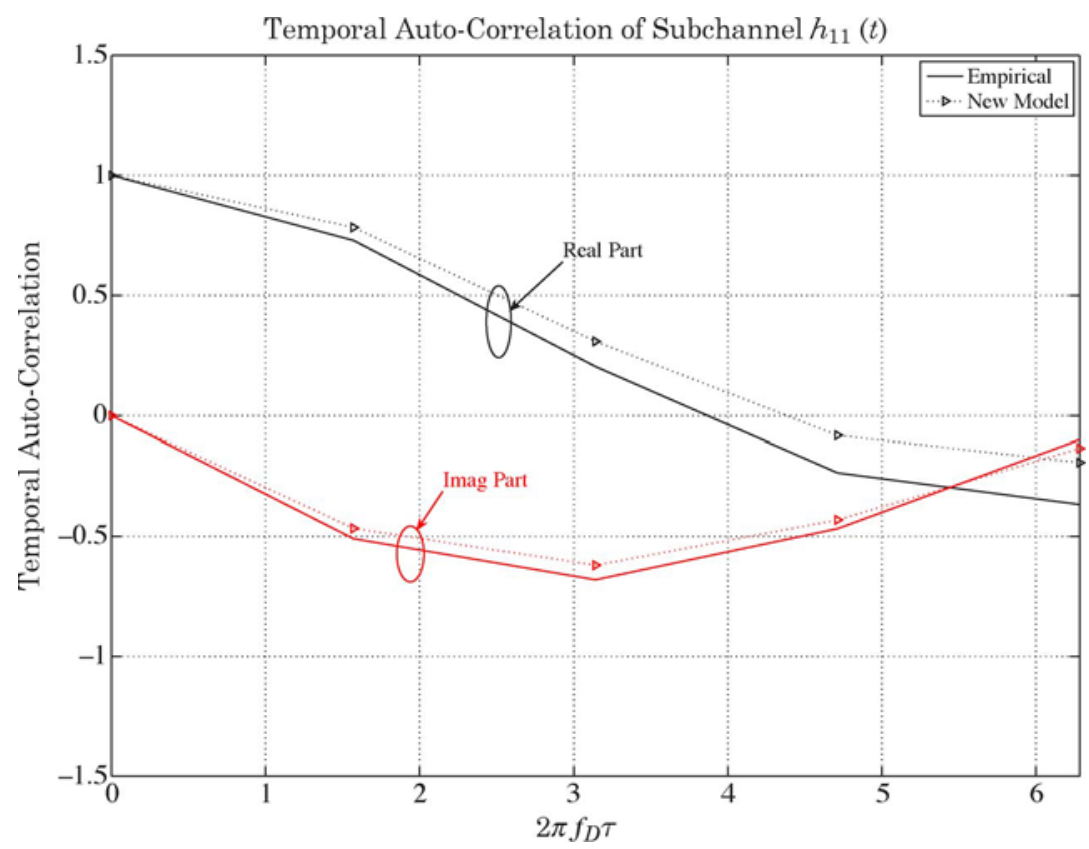

Fig. 6. Autocorrelation of $h_{11}(t)$, which is defined by $\mathbb{E}\left[h_{11}(t+\tau) h_{11}^{\star}(t)\right]$. New model and HUT data.

The theoretical LCR and AFD at the given threshold $r$ for and

Rayleigh fading are, respectively, given by [35]

$$
\bar{t}_{|h|}(r)=\frac{\sqrt{\pi}\left(e^{r^{2}}-1\right)}{r \sqrt{\mathcal{B}_{2}-\mathcal{B}_{1}^{2}}}
$$

$$
N_{|h|}(r)=\sqrt{\frac{\mathcal{B}_{2}-\mathcal{B}_{1}^{2}}{\pi}} r e^{-r^{2}}
$$

where $|h|$ denotes the amplitude of any subchannel. 


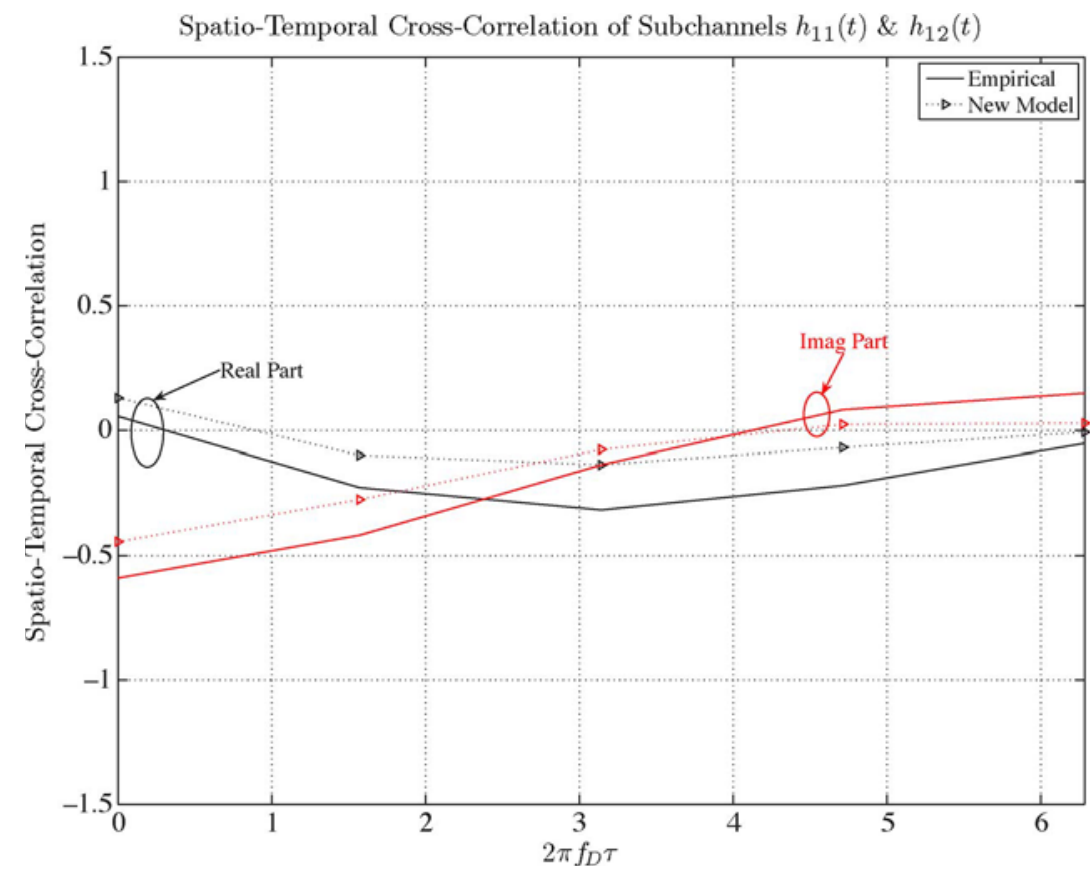

Fig. 7. STCC between $h_{11}(t)$ and $h_{12}(t)$, which is defined by $\mathbb{E}\left[h_{11}(t+\tau) h_{12}^{\star}(t)\right]$. New model and HUT data.

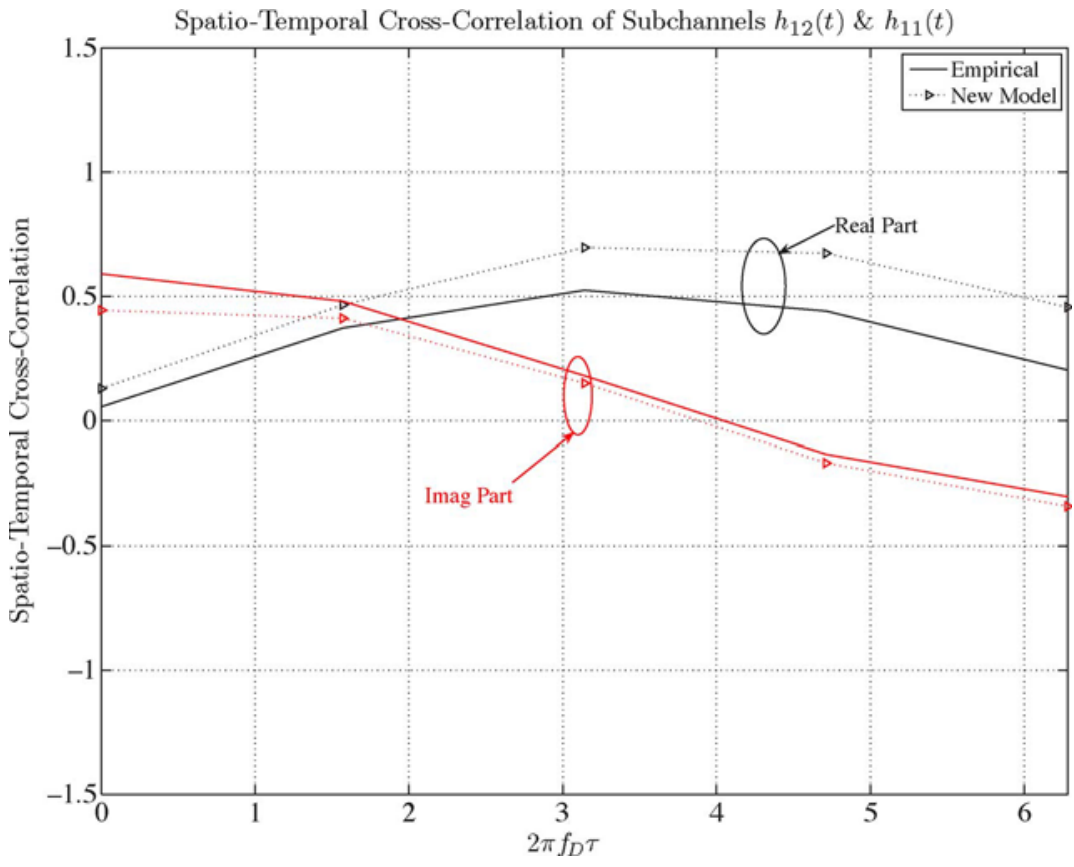

Fig. 8. STCC between $h_{12}(t)$ and $h_{11}(t)$, which is defined by $\mathbb{E}\left[h_{12}(t+\tau) h_{11}^{\star}(t)\right]$. New model and HUT data.

By plugging the estimated parameter set $\widehat{\boldsymbol{\Lambda}}_{\mathrm{HUT}}$ of Table I into (23) and (24), the LCR and AFD of the model are compared in Fig. 9 with the empirical LCR and AFD of $\left|h_{22}(t)\right|$ versus $r$. The close match for this and three other subchannels, which is not shown due to space limitation, verifies the ability of the model in capturing the dynamics of the channel.

\section{E. CDF of Instantaneous Mutual Information (IMI)}

In this section, we focus on the IMI under equal-power transmission to verify our proposed model. Assuming that the channel matrix $\mathbf{H}(t)$ is known at the receiver but not at the transmitter, with the MIMO channel characterized in (1), the IMI under equal-power transmission, in bits per second per hertz $(\mathrm{bps} / \mathrm{Hz})$, is given by [4]

$$
\mathcal{I}(t)=\log _{2} \operatorname{det}\left(\mathbf{I}_{N_{\mathrm{R}}}+\frac{P_{\mathrm{Tx}}}{N_{\mathrm{T}} P_{\text {Noise }}} \mathbf{H}(t) \mathbf{H}^{\dagger}(t)\right)
$$

where $\operatorname{det}(\cdot)$ is the matrix determinant. Note that at any time instant $t, \mathcal{I}(t)$ is a random variable, and its distribution depends 

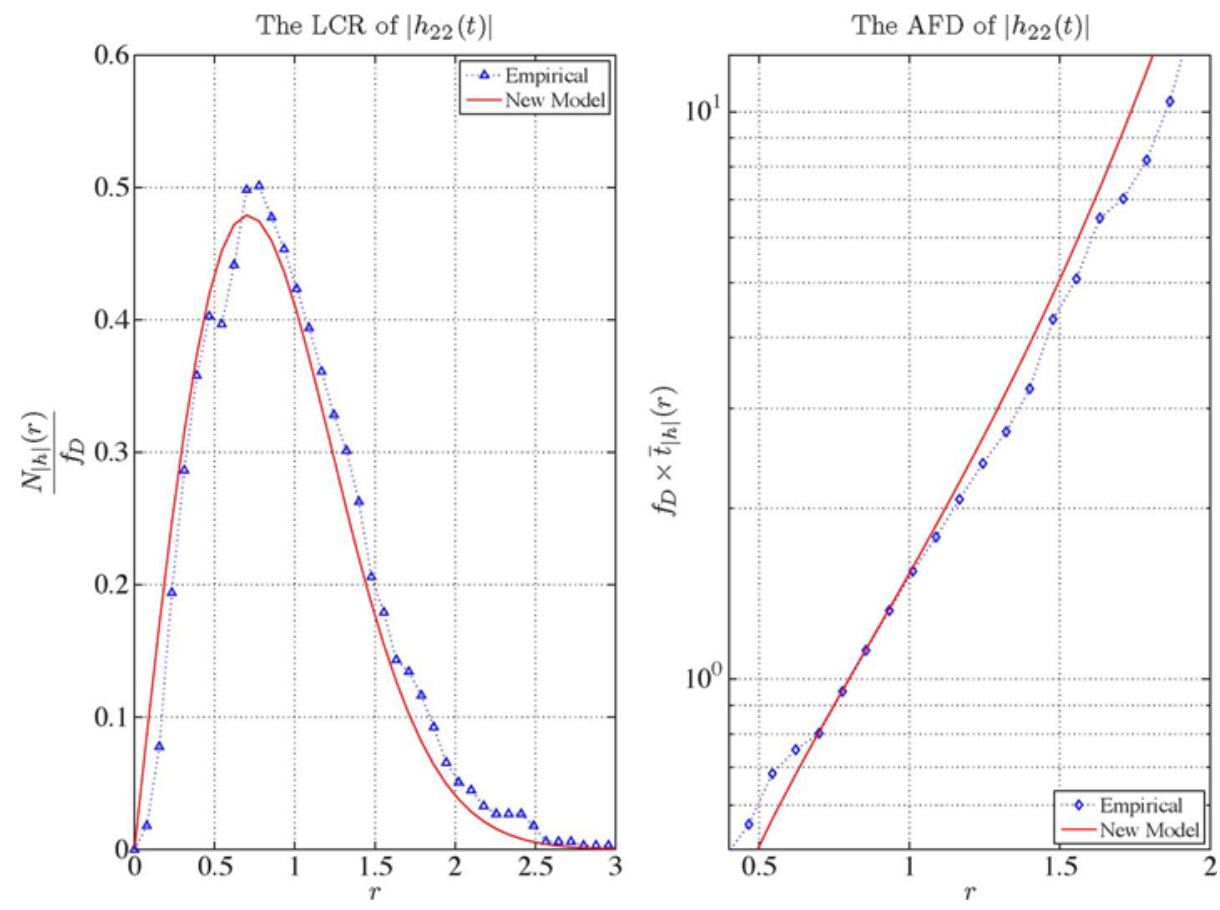

Fig. 9. LCR and AFD of $h_{22}(t)$. New model and HUT data.

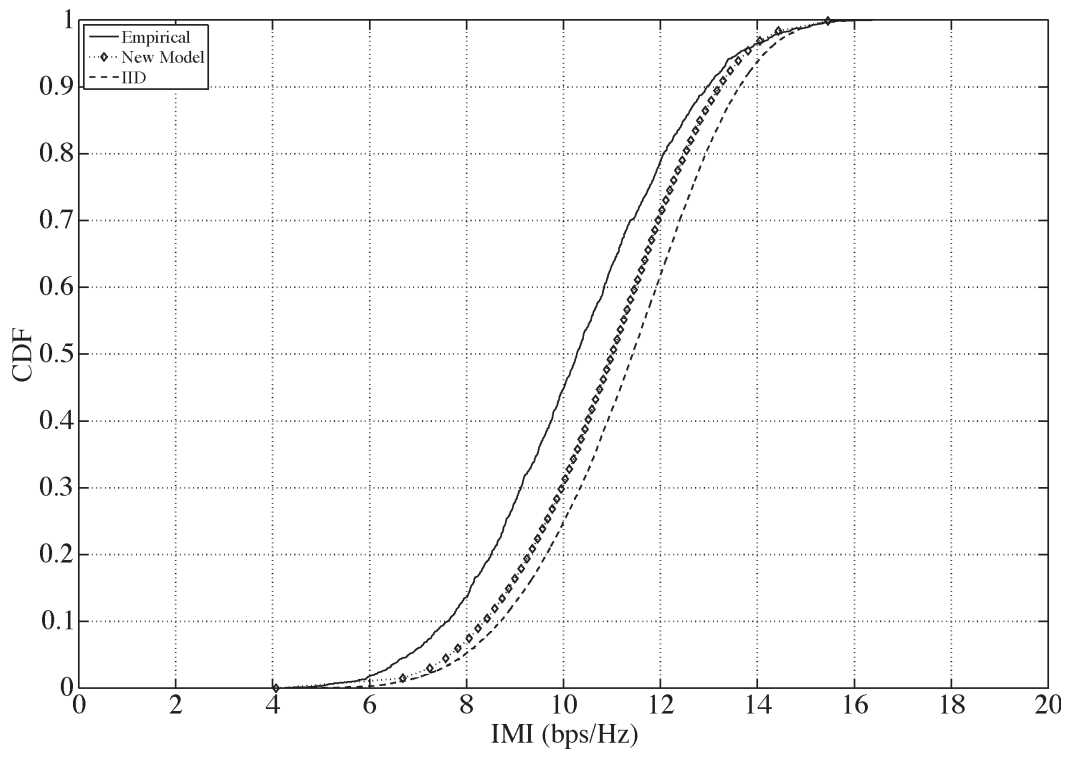

Fig. 10. Distributions of the IMI: HUT data.

on the distribution of the random matrix $\mathbf{H}(t) \mathbf{H}^{\dagger}(t)$. In this paper, we compare the empirical distribution of the MIMO IMI of the data, with the distribution predicted by our proposed model, where the $\mathrm{SNR}=P_{\mathrm{Tx}} / P_{\text {Noise }}$ is set to $20 \mathrm{~dB}$ in (25).

In Figs. 10 and 11, three different CDFs of IMI are plotted for the HUT and BYU data, respectively. The "empirical" IMI is calculated using (25) directly from the collected snapshots $\{\mathbf{H}(t)\}_{t=1}^{T}$, normalized according to (12). However, for each of the other IMIs, first, a zero-mean $N_{\mathrm{R}} \times N_{\mathrm{T}}$ Gaussian matrix $\mathbf{H}_{\text {IID }}$ with i.i.d. elements is generated for each $t$ such that $\mathbf{h}_{\text {IID }}=\operatorname{vec}\left(\mathbf{H}_{\text {IID }}\right)$ and $\mathbb{E}\left[\mathbf{h}_{\text {IID }} \mathbf{h}_{\text {IID }}^{\dagger}\right]=\mathbf{I}_{N_{\mathrm{R}} N_{\mathrm{T}}}$. The "IID" IMI CDF is obtained by inserting $\mathbf{H}_{\text {IID }}$ into (25). To obtain the "new model" IMI CDF, the following necessary steps are done.

1) For the HUT data, the correlated matrix-valued random process $\mathbf{H}(t)$ is generated using the spectral method [36] based on (7) and $\widehat{\Lambda}_{\mathrm{HUT}}$ of Table I and then plugged into (25).

2) For the BYU data, the colored Gaussian vector $\mathbf{h}\left(\widehat{\Lambda}_{\mathrm{BYU}}\right)$ is built according to $\mathbf{h}\left(\widehat{\boldsymbol{\Lambda}}_{\mathrm{BYU}}\right)=\sqrt{\mathbf{R}\left(\widehat{\boldsymbol{\Lambda}}_{\mathrm{BYU}}\right)} \mathbf{h}_{\mathrm{IID}}$ 


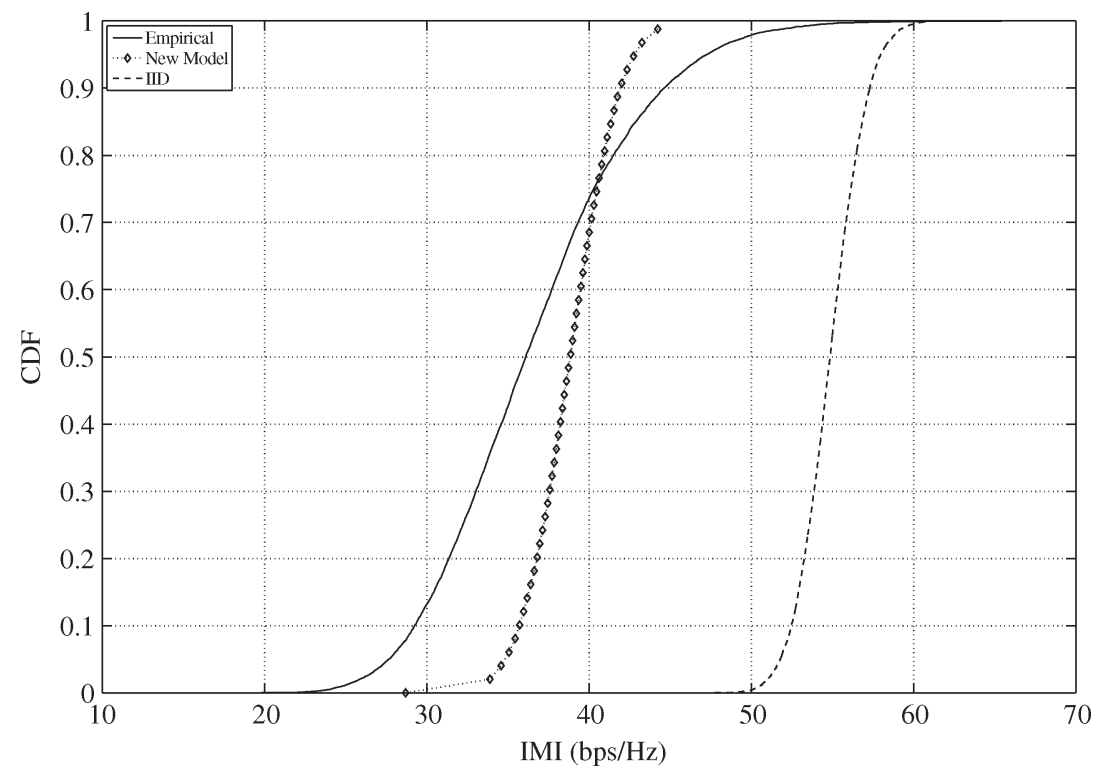

Fig. 11. Distributions of the IMI: BYU data.

where $\sqrt{\mathbf{R}\left(\widehat{\boldsymbol{\Lambda}}_{\mathrm{BYU}}\right)}$ is the Cholesky matrix of $\mathbf{R}\left(\widehat{\boldsymbol{\Lambda}}_{\mathrm{BYU}}\right)$, $\mathbf{R}(\boldsymbol{\Lambda})$ is defined in Section IV-B, and $\widehat{\Lambda}_{\mathrm{BYU}}$ is given in Table I. Then, the channel matrix $\mathbf{H}\left(\widehat{\boldsymbol{\Lambda}}_{\mathrm{BYU}}\right)$ is generated from $\mathbf{h}\left(\widehat{\Lambda}_{\mathrm{BYU}}\right)=\operatorname{vec}\left(\mathbf{H}\left(\widehat{\Lambda}_{\mathrm{BYU}}\right)\right)$ and $(25)$.

According to Figs. 10 and 11, the model fits the HUT data well. However, the match to the BYU data is not as good as the HUT data. This may be partly because the assumption $D \gg$ $\max \left(R^{\prime}, R\right)$ does not strictly hold for the scenarios where the BYU data were collected.

\section{CONCLUSION}

In this paper, a parametric statistical MIMO model is proposed where both the BS and the MS experience local scattering. The model yields a closed-form and mathematically tractable expression for the STCC between any two subchannels of a MIMO system with arbitrary transmit and receive array configurations. The model includes some key physical channel parameters such as the mean $\mathrm{AoD}$ and AoA, angle spreads, maximum Doppler frequency, sizes of the local scattering rings, and the relative contribution of BS and MS local scatterers.

The proposed model is general enough to include, as special cases, most existing correlation models such as the MIMO model of [5], Lee's spatio-temporal correlation model, and Clarke's classical spatial/temporal correlation model. The comparison of the model with outdoor and indoor collected data, in terms of spatial correlations, temporal autocorrelations, STCCs, LCRs, AFD, and the distribution of mutual information, has demonstrated the flexibility of the model in describing realworld propagation environments. The proposed model, which is supported by empirical observation, provides a useful channel characterization required for the efficient design and performance prediction of multiantenna transceivers.
APPENDIX A

\section{DERIVATION OF (5) AND (7)}

Based on the application of the law of cosines in appropriate triangles in Fig. 2, we obtain

$$
\begin{gathered}
\xi_{p x}^{\prime 2}=\delta_{p q}^{2} / 4+R^{\prime 2}-\delta_{p q} R^{\prime} \cos \left(\alpha_{p q}-x\right) \\
\xi_{q x}^{\prime 2}=\delta_{p q}^{2} / 4+R^{\prime 2}+\delta_{p q} R^{\prime} \cos \left(\alpha_{p q}-x\right) \\
\xi_{x l}^{\prime 2}=d_{l m}^{2} / 4+\xi_{x}^{\prime 2}-\xi_{x}^{\prime} d_{l m} \cos \left(v-\beta_{l m}\right) \\
\xi_{x m}^{\prime 2}=d_{l m}^{2} / 4+\xi_{x}^{\prime 2}+\xi_{x}^{\prime} d_{l m} \cos \left(v-\beta_{l m}\right) \\
\xi_{p y}^{2}=\delta_{p q}^{2} / 4+\xi_{y}^{2}-\xi_{y} \delta_{p q} \cos \left(\alpha_{p q}-w\right) \\
\xi_{q y}^{2}=\delta_{p q}^{2} / 4+\xi_{y}^{2}+\xi_{y} \delta_{p q} \cos \left(\alpha_{p q}-w\right) \\
\xi_{y l}^{2}=d_{l m}^{2} / 4+R^{2}-d_{l m} R \cos \left(y-\beta_{l m}\right) \\
\xi_{y m}^{2}=d_{l m}^{2} / 4+R^{2}+d_{l m} R \cos \left(y-\beta_{l m}\right)
\end{gathered}
$$

where, to simplify the notation, we use $x$ for $\phi_{k}^{\prime}, y$ for $\phi_{i}, v$ for $\varphi_{k}^{\prime}, w$ for $\varphi_{i}, \xi_{x}^{\prime}$ for $\xi_{k}^{\prime}$, and $\xi_{y}$ for $\xi_{i}$.

The following identity can be obtained by subtracting (26b) from (26a):

$$
\xi_{p x}^{\prime}-\xi_{q x}^{\prime}=\frac{-2 \delta_{p q} R^{\prime} \cos \left(\alpha_{p q}-x\right)}{\xi_{p x}^{\prime}+\xi_{q x}^{\prime}}
$$

With the assumption of $R^{\prime} \gg \delta_{p q}$, we have $\xi_{p x}^{\prime}+\xi_{q x}^{\prime} \approx 2 R^{\prime}$; therefore, (27) reduces to

$$
\xi_{p x}^{\prime}-\xi_{q x}^{\prime} \approx-\delta_{p q} \cos \left(\alpha_{p q}-x\right) .
$$

By applying the same reasoning to (26c)-(26h) and using the assumptions $D \gg \max \left(R^{\prime}, R\right)$ and $R \gg d_{l m}$, which imply 
$\xi_{x l}^{\prime}+\xi_{x m}^{\prime} \approx 2 \xi_{x}^{\prime}, \xi_{p y}+\xi_{q y} \approx 2 \xi_{y}$, and $\xi_{y l}+\xi_{y m} \approx 2 R$, we obtain the following approximations:

$$
\begin{aligned}
& \xi_{x l}^{\prime}-\xi_{x m}^{\prime} \approx-d_{l m} \cos \left(v-\beta_{l m}\right) \\
& \xi_{p y}-\xi_{q y} \approx-\delta_{p q} \cos \left(\alpha_{p q}-w\right) \\
& \xi_{y l}-\xi_{y m} \approx-d_{l m} \cos \left(y-\beta_{l m}\right) .
\end{aligned}
$$

Substitution of (28) and (29) into (4) results in the integral representation (5).

Now, we apply the law of sines to the triangles $O^{\prime} S_{k}^{\prime} O$ and $O^{\prime} S_{i} O$, respectively, to obtain the following identities:

$$
\begin{aligned}
& \frac{D}{\sin (v-x)}=\frac{R^{\prime}}{\sin (\pi-v)} \\
& \frac{D}{\sin (y-w)}=\frac{R}{\sin w} .
\end{aligned}
$$

From Fig. 2, it is clear that $\pi-v \leqslant \Delta^{\prime}, w \leqslant \Delta, \Delta^{\prime}=$ $\arcsin \left(R^{\prime} / D\right)$, and $\Delta=\arcsin (R / D)$. Based on the assumption $D \gg \max \left(R^{\prime}, R\right)$, we conclude that $\Delta^{\prime}$ and $\Delta$ and, consequently, $\pi-v$ and $w$ are small quantities. This observation, together with $\sin \epsilon \approx \epsilon$ when $\epsilon$ is small, allows us to derive the following approximations from (30a) and (30b):

$$
\begin{aligned}
v & \approx \pi-\Delta^{\prime} \sin x \\
w & \approx \Delta \sin y .
\end{aligned}
$$

Furthermore, using $\sin \epsilon \approx \epsilon$ and $\cos \epsilon \approx 1$ when $\epsilon$ is small, together with (31a) and (31b), $\cos \left(v-\beta_{l m}\right)$ and $\cos \left(\alpha_{p q}-w\right)$ can be approximated as

$$
\begin{aligned}
\cos \left(v-\beta_{l m}\right) & \approx-\cos \beta_{l m}+\Delta^{\prime} \sin \beta_{l m} \sin x \\
\cos \left(\alpha_{p q}-w\right) & \approx \cos \alpha_{p q}+\Delta \sin \alpha_{p q} \sin y .
\end{aligned}
$$

By plugging (6b), (6a), (32a), and (32b) into (5) and calculating the two integrals according to [37, 3.338-4]

$$
\int_{-\pi}^{\pi} \exp (x \sin \theta+y \cos \theta) d \theta=2 \pi I_{0}\left(\sqrt{x^{2}+y^{2}}\right)
$$

the general STCC in (7) can be obtained after some algebraic manipulations.

\section{APPENDIX B HUT DATA}

Fig. 12 shows the layout of the location where the HUT data are collected. The $2 \times 2$ measurement setup and four subchannels are shown in Fig. 13(a), whereas the configuration of the Rx array is presented in Fig. 13(b). The Tx and Rx element spacings are given by $\delta_{12}=\lambda$ and $d_{12}=2.785 \lambda$, respectively. The mobile speed is $0.4 \mathrm{~m} / \mathrm{s}$, which, with the $2.154-\mathrm{GHz}$ carrier frequency, results in $f_{D}=\nu / \lambda=2.872 \mathrm{~Hz}$. There are $T=1342$ snapshots of the channel matrix $\mathbf{H}(t)$,

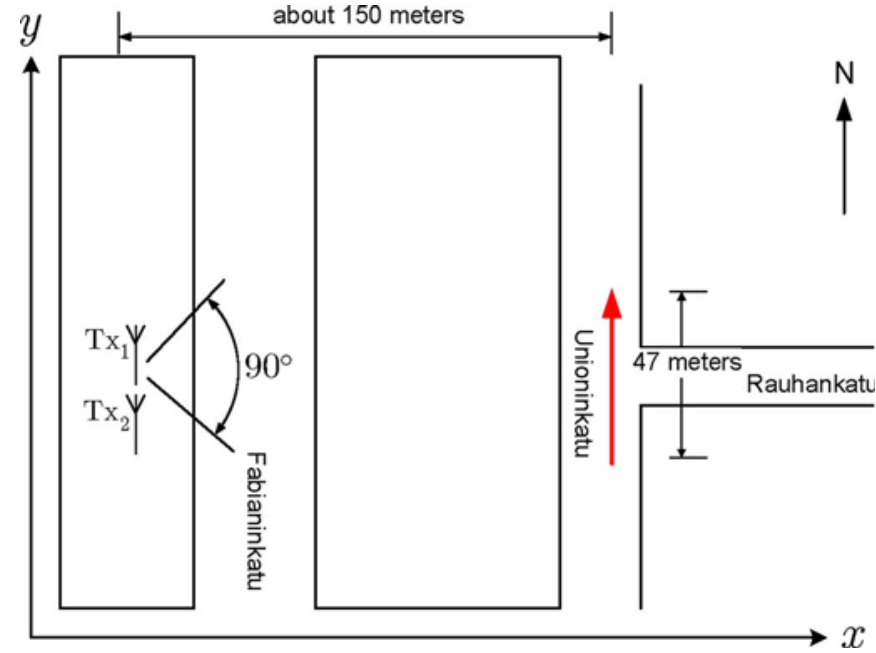

Fig. 12. Layout of the environment campaign of the HUT data.

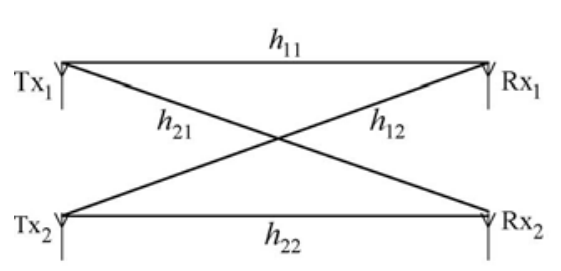

(a)

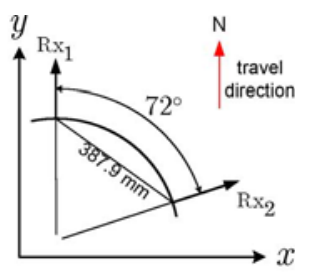

(b)
Fig. 13. Measurement setup for the HUT data. (a) $2 \times 2$ sysrem. (b) Receiver array.

and any two adjacent snapshots are separated by $\lambda / 4$ in space, which is equivalent to $87.05 \mathrm{~ms}$ in time. Further details of the data and measurement setup can be found in [21] and [22]. The pathloss is removed from the data by least-square fitting of a tenth order polynomial in the decibel domain, and the power normalization is performed according to (12).

\section{APPENDIX C BYU DATA}

For the data collected on November 7, 2000, at BYU, the room layout is shown in Fig. 14. Both the Tx and Rx arrays are linear, each with ten monopole elements and $\lambda / 4$ spacing at the carrier frequency of $2.42 \mathrm{GHz}$. The Tx array was placed at four different locations in room 484, whereas the Rx array was placed at six different locations in room 400 . There are 24 $\mathrm{Tx}-\mathrm{Rx}$ location configurations, 20 data sets per location, and 124 snapshots of the $10 \times 10$ channel matrix per data set. Power normalization of each data set was done according to

$$
\begin{aligned}
\tilde{h}_{\mathrm{loc}, \text { set }, l p}(t)= & \frac{h_{\mathrm{loc}, \text { set }, l p}(t)-\widehat{m}_{\mathrm{loc}, \mathrm{set}, l p}}{\widehat{\sigma}_{\mathrm{loc}, \mathrm{set}, l p}} \\
& \operatorname{set} \in[1,20], l, p \in[1,10], t \in[1,124]
\end{aligned}
$$

where loc is the location index $\left\{1 \_1, \ldots, 1 \_6,2 \_1, \ldots, 2 \_6\right.$, $\left.3 \_1, \ldots, 3 \_6,4 \_1, \ldots, 4 \_6\right\}$, set is the data set index at each location, and $\widehat{m}_{\text {loc,set, } l p}$ and $\widehat{\sigma}_{\text {loc,set }, l p}^{2}$ are the estimated mean and variance of the subchannel $h_{l p}(t)$ in the set ${ }^{\text {th }}$ data set at the 

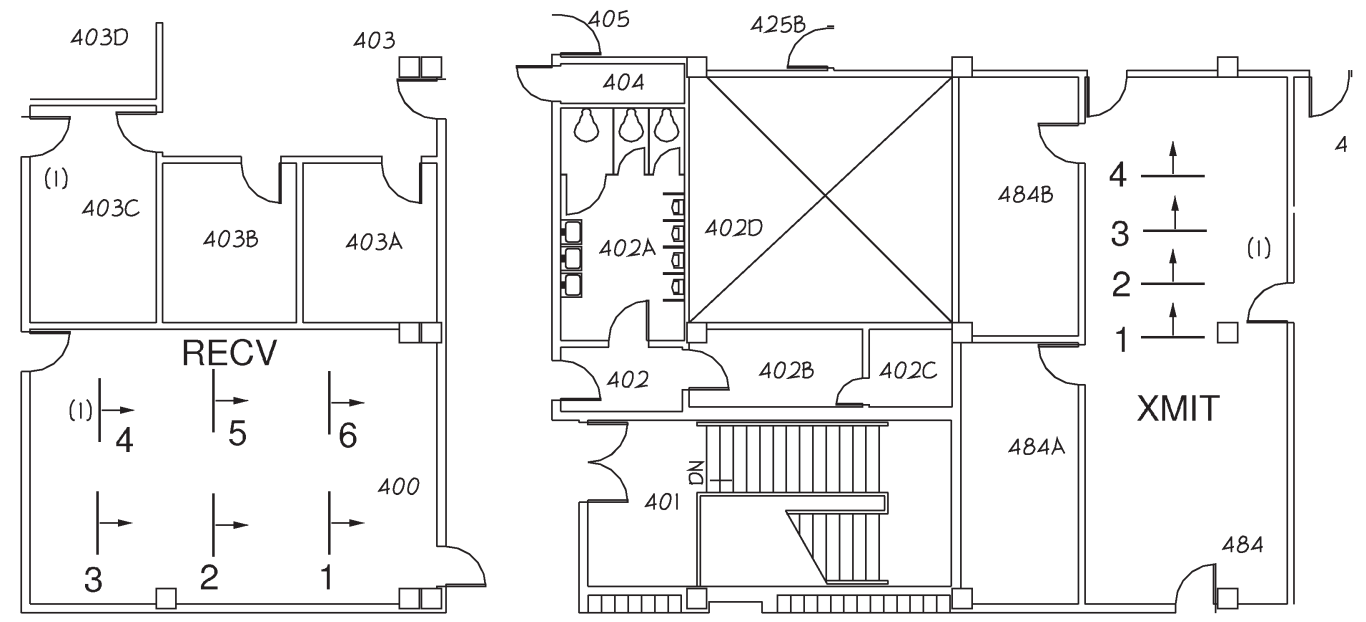

Fig. 14. Room layout where the BYU data are collected. In the figure, “ $\uparrow$ ” denotes ten antenna elements that are numbered as $\frac{\uparrow}{1, \ldots, 10}$.

loc ${ }^{\text {th }}$ location. Because of the fixed Tx and Rx and stationary environment, we have $f_{D}=0 \mathrm{~Hz}$. More measurement information can be found in [33, ch. 6 and 7] and [34], where this data set is labeled as " $10 \times 10(a)$."

\section{ACKNOWLEDGMENT}

The authors would like to thank the anonymous reviewers for their careful reviews of this paper. Their comments have certainly improved the quality of this paper.

\section{REFERENCES}

[1] S. Wang, K. Raghukumar, A. Abdi, J. Wallace, and M. Jensen, "Indoor MIMO channels: A parametric correlation model and experimental results," in Proc. IEEE Sarnoff Symp., Princeton, NJ, 2004, pp. 1-5.

[2] S. Wang, A. Abdi, J. Salo, H. El-Sallabi, and P. Vainikainen, "A timevarying MIMO channel model: Theory and measurements," in Proc. IEEE Int. Workshop Signal Process. Adv. Wireless Commun., New York, 2005, pp. 343-347.

[3] G. J. Foschini and M. J. Gans, "On limits of wireless communications in a fading environment when using multiple antennas," Wirel. Pers. Commun., vol. 6, no. 3, pp. 311-335, Mar. 1998.

[4] İ. E. Telatar, "Capacity of multi-antenna Gaussian channels," Eur. Trans. Telecommun., vol. 10, no. 6, pp. 585-595, Nov./Dec. 1999.

[5] A. Abdi and M. Kaveh, "A space-time correlation model for multielement antenna systems in mobile fading channels," IEEE J. Sel. Areas Commun., vol. 20, no. 3, pp. 550-560, Apr. 2002.

[6] P. J. Smith, S. Roy, and M. Shafi, "Capacity of MIMO systems with semicorrelated flat fading," IEEE Trans. Inf. Theory, vol. 49, no. 10, pp. 2781-2788, Oct. 2003 .

[7] M. Chiani, M. Z. Win, and A. Zanella, "On the capacity of spatially correlated MIMO Rayleigh-fading channels," IEEE Trans. Inf. Theory, vol. 49, no. 10, pp. 2363-2371, Oct. 2003.

[8] A. Goldsmith, S. A. Jafar, N. Jindal, and S. Vishwanath, "Capacity limits of MIMO channels," IEEE J. Sel. Areas Commun., vol. 21, no. 5, pp. 684-702, Jun. 2003.

[9] D. P. Liu, Q. T. Zhang, and Q. Chen, "Structures and performance of noncoherent receivers for unitary space-time modulation on correlated fast-fading channels," IEEE Trans. Veh. Technol., vol. 53, no. 4, pp. 11161125, Jul. 2004

[10] G. Femenias, "BER performance of linear STBC from orthogonal designs over MIMO correlated Nakagami-m fading channels," IEEE Trans. Veh. Technol., vol. 53, no. 2, pp. 307-317, Mar. 2004.

[11] V. K. Nguyen and L. B. White, "Joint space-time trellis decoding and channel estimation in correlated fading channels," IEEE Signal Process. Lett., vol. 11, no. 7, pp. 633-636, Jul. 2004.

[12] M. Jankiraman, Space-Time Codes and MIMO Systems. Boston, MA: Artech House, 2004.
[13] K. Yu and B. Ottersten, "Models for MIMO propagation channels: A review," Wirel. Commun. Mob. Comput., vol. 2, no. 7, pp. 653-666, Nov. 2002.

[14] A. Abdi, "Stochastic modeling and simulation of multiple-input multipleoutput channels: A unified approach," in Proc. IEEE Int. Symp. Antennas Propag., Monterey, CA, 2004, pp. 3673-3676.

[15] A. F. Molisch, "A generic model for MIMO wireless propagation channels in macro- and microcells," IEEE Trans. Signal Process., vol. 52, no. 1, pp. 61-71, Jan. 2004

[16] M. A. Jensen and J. W. Wallace, "A review of antennas and propagation for MIMO wireless communications," IEEE Trans. Antennas Propag., vol. 52, no. 11, pp. 2810-2824, Nov. 2004.

[17] M. Steinbauer, D. Hampicke, G. Sommerkorn, A. Schneider, A. F. Molisch, R. Thoma, and E. Bonek, "Array measurement of the double-directional mobile radio channel," in Proc. IEEE Veh. Technol. Conf., Tokyo, Japan, 2000, pp. 1656-1662.

[18] M. Steinbauer, A. F. Molisch, and E. Bonek, "The double-directional radio channel," IEEE Antennas Propag. Mag., vol. 43, no. 4, pp. 51-63, Aug. 2001.

[19] S. Savazzi, O. Simeone, and U. Spagnolini, "Optimal design of linear arrays in a TDMA cellular system with Gaussian interference," in Proc. IEEE Int. Workshop Signal Process. Adv. Wireless Commun., New York, 2005, pp. 485-489.

[20] D. Gesbert, H. Bolcskei, D. A. Gore, and A. J. Paulraj, "Outdoor MIMO wireless channels: Models and performance prediction," IEEE Trans. Commun., vol. 50, no. 12, pp. 1926-1934, Dec. 2002.

[21] K. Sulonen, P. Suvikunnas, J. Kivinen, L. Vuokko, and P. Vainikainen, "Study of different mechanisms providing gain in MIMO systems," in Proc. IEEE Veh. Technol. Conf., Orlando, FL, 2003, pp. 352-356.

[22] K. Kalliola, H. Laitinen, L. I. Vaskelainen, and P. Vainikainen, "Realtime 3-D spatial-temporal dual-polarized measurement of wideband radio channel at mobile station," IEEE Trans. Instrum. Meas., vol. 49, no. 2, pp. 439-448, Apr. 2000.

[23] J. W. Wallace and M. A. Jensen, "Measured characteristics of the MIMO wireless channel," in Proc. IEEE Veh. Technol. Conf., Atlantic City, NJ, 2001, pp. 2038-2042.

[24] Narrowband (30 kHz With the Carrier Frequency of $2.42 \mathrm{GHz}$ ) MIMO data collected on Nov. 7, 2000. [Online]. Available: http://ee.byu.edu/wireless/

[25] A. Abdi, J. A. Barger, and M. Kaveh, "A parametric model for the distribution of the angle of arrival and the associated correlation function and power spectrum at the mobile station," IEEE Trans. Veh. Technol., vol. 51, no. 3, pp. 425-434, May 2002.

[26] G. L. Stüber, Principles of Mobile Communications, 2nd ed. Boston, MA: Kluwer, 2000.

[27] T. L. Fulghum, K. J. Molnar, and A. Duel-Hallen, "The Jakes fading model for antenna arrays incorporating azimuth spread," IEEE Trans. Veh. Technol., vol. 51, no. 5, pp. 968-977, Sep. 2002.

[28] J. W. Wallace and M. A. Jensen, "Modeling the indoor MIMO wireless channel," IEEE Trans. Antennas Propag., vol. 50, no. 5, pp. 591-599, May 2002. 
[29] K. Yu, M. Bengtsson, B. Ottersten, D. McNamara, P. Karlsson, and M. Beach, "Second order statistics of NLOS indoor MIMO channels based on $5.2 \mathrm{GHz}$ measurements," in Proc. IEEE Global Telecommun. Conf., San Antonio, TX, 2001, pp. 156-160.

[30] C. B. Ribeiro, E. Ollila, and V. Koivunen, "Propagation parameter estimation in MIMO systems using mixture of angular distributions model," in Proc. IEEE Int. Conf. Acoust., Speech, Signal Process., Philadelphia, PA, 2005, pp. 18-23.

[31] N. K. Bose and K. J. Boo, "Asymptotic eigenvalue distribution of blocktoeplitz matrices," IEEE Trans. Inf. Theory, vol. 44, no. 2, pp. 858-861, Mar. 1998.

[32] A. Papoulis and S. U. Pillai, Probability, Random Variables and Stochastic Processes, 4th ed. New York: McGraw-Hill, 2001.

[33] J. W. Wallace, "Modeling electromagnetic wave propagation in electrically large structures," Ph.D. dissertation, Brigham Young Univ., Provo, UT, 2002.

[34] J. W. Wallace, M. A. Jensen, A. L. Swindlehurst, and B. D. Jeffs, "Experimental characterization of the MIMO wireless channel: Data acquisition and analysis," IEEE Trans. Wireless Commun., vol. 2, no. 2, pp. 335-343, Mar. 2003.

[35] A. Abdi, K. Wills, H. A. Barger, M. S. Alouini, and M. Kaveh, "Comparison of the level crossing rate and average fade duration of Rayleigh, Rice, and Nakagami fading models with mobile channel data," in Proc. IEEE Veh. Technol. Conf., Boston, MA, 2000, pp. 1850-1857.

[36] K. Acolatse and A. Abdi, "Efficient simulation of space-time correlated MIMO mobile fading channels," in Proc. IEEE Veh. Technol. Conf., Orlando, FL, 2003, pp. 652-656.

[37] I. S. Gradshteyn, I. M. Ryzhik, and A. Jeffrey, Eds., Table of Integrals, Series, and Products, 5th ed. San Diego, CA: Academic, 1994.

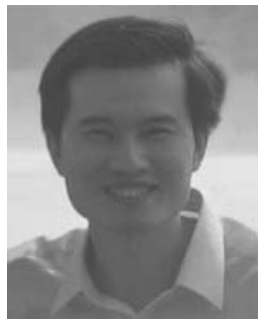

Shuangquan Wang (S'00) received the B.S. degree in computer engineering from Xi' an Institute of Posts and Telecommunications, Xi' an, China, in 1998, the M.S. degree in electrical engineering from Beijing University of Posts and Telecommunications (BUPT), Beijing, China, in 2001, and the Ph.D. degree in electrical engineering from the New Jersey Institute of Technology, Newark, in 2006.

From 2001 to 2002, he worked on time division synchronous code division multiple access (TD-SCDMA) with Siemens Ltd., China, Beijing. Currently, he is with NEC Laboratories America, Inc., Princeton, NJ. His current research interests include multiple-input multiple-output (MIMO) channel modeling, estimation and its second-order statistics, random matrix theory and its application in wireless MIMO communications, multiuser communications, and space-time transceiver design in MIMO orthogonal frequency-divisionmultiplexing (OFDM) systems.

Dr. Wang has won several awards for his research contributions. These include the BUPT EI index prize in 2005, the 2005 New Jersey Inventors Hall of Fame Graduate Student Award, First Place in the Graduate Poster Contest, IEEE GLOBECOM'05, and the Hashimoto Prize.

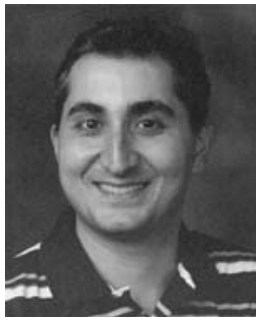

Ali Abdi (S'98-M'01) received the Ph.D. degree in electrical engineering from the University of Minnesota, Minneapolis, in 2001.

He has been with the Department of Electrical and Computer Engineering, New Jersey Institute of Technology, Newark, since 2001, as an Assistant Professor. His current research interests include estimation and characterization of wireless fading channels, digital communication in terrestrial and underwater channels, blind modulation recognition and parameter-estimation techniques, spacetime processing, and interference cancellation.

Dr. Abdi is an Associate Editor for the IEEE TRANSACTIONS ON Vehicular TECHNOLOGY.

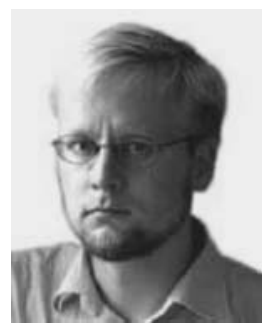

Jari Salo received the Master of Science, Licentiate of Science, and Doctor of Science degrees from Helsinki University of Technology, Espoo, Finland, in 2000, 2003, and 2006, respectively.

From 2001 to 2002, he was with Bitville Oy. $\mathrm{He}$ is currently with the European Communications Engineering Ltd., Espoo, where he is a Network Planning Engineer.

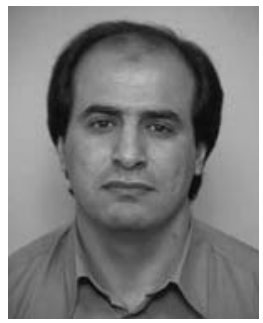

Hassan M. El-Sallabi (S'99-M'03) received the B.Sc. (with honors) and M.Sc. degrees in electrical engineering from Garyounis University (GU), Benghazi, Libya, and the Licentiate and D.Sc. (with distinction) degrees from Helsinki University of Technology (TKK), Espoo, Finland.

He worked as a Telecommunication Engineer in a general electric company, Benghazi, where he held several positions. He also worked as an Assistant Lecturer with the Faculty of Engineering, GU. He held positions as a Senior Research Engineer and a Project Manager with the Radio Laboratory at TKK from September 2003 to September 2005. He also served as a Task Leader with the WINNER European Commission project from January 2004 to the end of September 2005. Since October 2005, he has been a Postdoctoral Researcher with the Information Systems Laboratory, Stanford University, Stanford, CA. His research interests include physical and stochastic channel modeling for wireless communications, diffraction theory, wireless system designs, time reversal communications, and multiantenna techniques. He has published more than 50 journal and conference papers, contributed to the organization of various international conferences as a member of the Technical Program Committee and as a Session Chairman, and served as a Reviewer for many international journals.

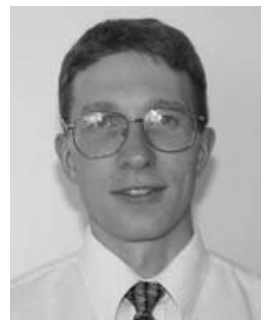

Jon W. Wallace (S'99-M'03) received the B.S. (summa cum laude) and Ph.D. degrees in electrical engineering from Brigham Young University (BYU), Provo, UT, in 1997 and 2002, respectively.

From 1995 to 1997, he was an Associate with Novell, Inc., Provo. In 1997, he was a member of the Technical Staff of Lucent Technologies, Denver, CO. He received the National Science Foundation Graduate Fellowship in 1998 and worked as a Graduate Research Assistant at BYU until 2002. From 2002 to 2003, he was with the Mobile Communications Group, Vienna University of Technology, Vienna, Austria, pursuing collaborative research in the area of wireless channel measurement and modeling. From 2003 to 2006, he was a Research Associate with the BYU Wireless Communications Laboratory, developing platforms for wideband multiple-input multiple-output (MIMO) channel sounding and real-time space-time coding. $\mathrm{He}$ is currently an Assistant Professor with the International University Bremen, Bremen, Germany. His research interests include wireless channel sounding and modeling, real-time MIMO implementation, optical device modeling, and remote sensing. 


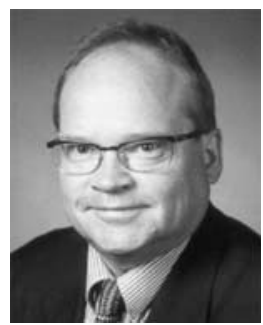

Pertti Vainikainen (M'91) received the Master of Science in Technology, Licentiate of Science in Technology, and Doctor of Science in Technology degrees from Helsinki University of Technology (TKK), Espoo, Finland, in 1982, 1989, and 1991, respectively.

From 1992 to 1993, he was an Acting Professor of radio engineering, since 1993, an Associate Professor of radio engineering, and since 1998, a Professor of radio engineering with the Radio Laboratory, TKK. From 1993 to 1997, he was the Director of the Institute of Radio Communications of TKK, a Visiting Professor in 2000 at Aalborg University, Aalborg, Denmark, and in 2006, he visited the University of Nice, Nice, France. His main fields of research interest are antennas and propagation in radio communications and industrial measurement applications of radio waves. He is the Author or Coauthor of six books or book chapters and about 250 refereed international journal or conference publications and the holder of seven patents.

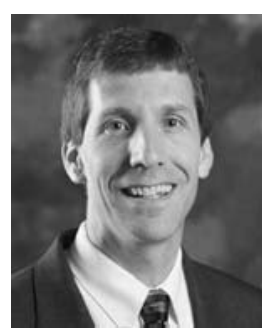

Michael A. Jensen (S'93-M'95-SM'01) received the B.S. (summa cum laude) and M.S. degrees in electrical engineering from Brigham Young University (BYU), Provo, UT, in 1990 and 1991, respectively, and the Ph.D. degree in electrical engineering from the University of California, Los Angeles (UCLA), in 1994.

From 1989 to 1991, he was a Graduate Research Assistant with the Lasers and Optics Laboratory at BYU. In 1990, he received a National Science Foundation Graduate Fellowship. From 1991 to 1994, he was a Graduate Student Researcher with the Antenna Laboratory, UCLA. Since 1994, he has been with the Department of Electrical and Computer Engineering, BYU, where he is currently a Professor and the Department Chair. His main research interests include antennas and propagation for personal communications, microwave circuit design, radar remote sensing, numerical electromagnetics, and optical fiber communications.

Dr. Jensen is a member of Eta Kappa Nu and Tau Beta Pi. He currently serves as a member of the Administrative Committee and the Joint Meetings Committee for the IEEE Antennas and Propagation Society and as an Associate Editor for the IEEE TRANSACTIONS ON ANTENNAS AND PROPAGATION. He has also served the society as Vice-Chair and Technical Program Chair for several symposia. He received the $\mathrm{H}$. A. Wheeler Paper Award of the IEEE Transactions on Antennas and Propagation in 2002 and the Best Student Paper Award at the 1994 IEEE International Symposium on Antennas and Propagation. 